\title{
Hilbert Modular Polynomials
}

\author{
Chloe Martindale \\ Department of Mathematics and Computer Science, \\ Technische Universiteit Eindhoven, \\ P.O. Box 513, 5600 MB Eindhoven, The Netherlands \\ chloemartindale@gmail.com
}

\begin{abstract}
We present an algorithm to compute a higher dimensional analogue of modular polynomials. This higher dimensional analogue, the 'set of Hilbert modular polynomials', concerns cyclic isogenies of principally polarised abelian varieties with maximal real multiplication by a fixed totally real number field $K_{0}$. In the 2-dimensional case with $K_{0}=\mathbb{Q}(\sqrt{5})$ we also provide an implementation together with some optimisations specific to this case. We also explain applications of this algorithm to point counting, walking on isogeny graphs, and computing class polynomials.
\end{abstract}

Keywords: Hilbert modular polynomials, cyclic isogenies, abelian varieties, genus two, maximal real multiplication

\section{Introduction}

The modular polynomial for elliptic curves of prime level $\ell$ is an irreducible polynomial $\Phi_{\ell}(X, Y) \in \mathbb{Z}[X, Y]$ which, for every pair of $\ell$-isogenous elliptic curves $E$ and $E^{\prime}$, satisfies

$$
\Phi_{\ell}\left(j(E), j\left(E^{\prime}\right)\right)=0,
$$

where $j(E)$ is the $j$-invariant of the elliptic curve $E$. Examples of these modular polynomials can be found for example on Sutherland's website [31. One of the reasons that modular polynomials interest us is that given the $j$-invariant of an elliptic curve $E$ over a field $k$, we can find the $j$-invariants of all those elliptic curves that are $\ell$-isogenous to it by computing the roots of $\Phi_{\ell}(j(E), Y) \in k[Y]$. In this article, we describe an analogue of the modular polynomial for principally polarised abelian varieties of dimension $g$ with real multiplication, which we call a set of Hilbert modular polynomials. This set of Hilbert modular polynomials is computed using Algorithm 6.3, one case of which is implemented in Magma 4] and is available at www.martindale.info/research. Exactly as for elliptic curves, the Hilbert modular polynomials can be used to study isogenies with cyclic kernel (that preserve the polarisation and the real multiplication in a 
natural way). Note that these isogenies are not $(\ell, \ldots, \ell)$-isogenies-an $(\ell, \ldots, \ell)$ isogeny can (under certain conditions) be decomposed into the ' $\mu$-isogenies' (c.f. Definition 2.2 studied in this article.

There are few different proposals in the literature for generalising modular polynomials for $\ell$-isogenies of elliptic curves to $(\ell, \ell)$-isogenies of principally polarised abelian surfaces. Dupont [10] gave an analogue that allows us to compute $(\ell, \ell)$-isogenous principally polarised abelian surfaces (not taking into account real multiplication); there were also follow-up works by Bröker and Lauter [6] and Milio 23]. These papers use the 'Siegel moduli space' interpretation where we propose to use the 'Hilbert moduli space' interpretation. The advantage of working in the Hilbert setting is that the coefficients and degrees of the polynomials are much more manageable than in the Siegel setting, making it possible to compute modular polynomials for higher prime levels than previously.

We argue that cyclic ' $\mu$-isogenies' are, in the case of maximal real multiplication, a more natural generalisation of $\ell$-isogenies of elliptic curves than $(\ell, \ldots, \ell)$-isogenies. The applications presented in Section 8.2 refer to 'walking on isogeny graphs'. There are many algorithms for elliptic curves that use the volcano structure of the isogeny graph of elliptic curves, for example [32] or [8], or the endomorphism ring computation algorithm of $[18$. The $(\ell, \ldots, \ell)$-isogeny graph of principally polarised abelian surfaces does not in general have a volcano structure, but an isogeny graph of the cyclic ' $\mu$-isogenies' studied in this article does have a volcano structure, see [20, Chapter 3]. Hence, any algorithm for elliptic curves that uses the ability to walk on the volcano isogeny graph immediately generalises to principally polarised abelian varieties with maximal real multiplication using the modular polynomials presented in this paper (see Section 8.2 for some more details, including computational feasability). Furthermore, any application that requires you to compute a composition of isogenies between two specific principally polarised abelian varieties with maximal real multiplication may not be possible with only $(\ell, \ldots, \ell)$-isogenies.

Remark 1.1. In his $\mathrm{PhD}$ thesis, Milio 22 independently described an algorithm to compute Hilbert modular polynomials for a slightly different type of cyclic isogenies (they do not necessarily preserve the embedding of the maximal real order into the endomorphism ring), with a focus on dimension two. The methods of computation are in Milio's thesis and this work are also fundamentally different: while we use linear algebra on the Fourier coefficients of the Hilbert modular form (analogously to Enge's method [11, Section 2.2] in genus 1), he uses evaluation/interpolation (analogously to Enge's method [11, Section 3] in genus 1). The advantage of his method is speed: for dimension 2 his algorithm is quasilinear. Our method on the other hand works (theoretically) in any dimension and for any modular invariant for which one can compute the Fourier coefficients. Milio and Robert 24] have also independently written a follow up paper presenting other, fundamentally different methods of computing Hilbert modular polynomials.

The modular polynomial for elliptic curves of level $\ell$ parametrises $\ell$-isogenies of elliptic curves (for $\ell$ prime) and is defined using the $j$-invariant. To generalise the 
modular polynomial to a Hilbert modular setting, we first fix a totally real number field $K_{0}$ of degree $g$ over $\mathbb{Q}$, and we write $\mathcal{O}_{K_{0}}$ for its maximal order. We then need to replace $j$ by an 'isomorphism invariant' for objects $(\mathrm{A}, \xi, \iota) \in \mathbf{P O r d}_{\mathbb{C}, K_{0}}$, the category of principally polarised complex abelian $g$-folds $(\mathrm{A}, \xi)$ with an appropriate embedding $\iota: \mathcal{O}_{K_{0}} \hookrightarrow \operatorname{End}(\mathrm{A})$ (see Definition 2.1 for the formal definition). Let $\bar{V}$ be the Hilbert modular variety for $\operatorname{SL}\left(\mathcal{O}_{K_{0}} \oplus \mathcal{O}_{K_{0}}^{\vee}\right)$, as in Definition 2.13, where $\mathcal{O}_{K_{0}}^{\vee}$ is the trace dual of $\mathcal{O}_{K_{0}}$. We denote by $\mathcal{M}_{K_{0}}(\mathbb{Z})$ the ring of Hilbert modular forms with coefficients in $\mathbb{Z}$ (c.f. Definition 2.10), and we write $\mathbb{Q}\left(\mathcal{M}_{K_{0}}(\mathbb{Z})\right)$ for the field of quotients of modular forms in $\mathcal{M}_{K_{0}}(\mathbb{Z})$ of equal weight. We will see in Section 3 that for some $d \in \mathbb{Z}$, there exist $d$ Hilbert modular functions

$$
J_{1}, \ldots, J_{d} \in \mathbb{Q}\left(\mathcal{M}_{K_{0}}(\mathbb{Z})\right),
$$

such that the function field of $\bar{V}$ is $\mathbb{C}\left(J_{1}, \ldots, J_{d}\right)$, and for such $J_{1}, \ldots, J_{d}$, there exists a Zariski-open affine subvariety $U$ of $\bar{V}$ such that the rational map

$$
\left(J_{1}, \ldots, J_{d}\right): U--\rightarrow \mathbb{A}_{\mathbb{C}}^{d}
$$

is an injective morphism.

Definition 1.2. A $d$-tuple of $\mathbb{Q}$-linearly independant, non-constant Hilbert modular functions $\left(J_{1}, \ldots, J_{d}\right) \in \mathbb{Q}\left(\mathcal{M}_{K_{0}}(\mathbb{Z})\right)^{\times d}$ such that

$$
\mathbb{C}(\bar{V})=\mathbb{C}\left(J_{1}, \ldots, J_{d}\right)
$$

is a choice of $R M$ isomorphism invariants for $K_{0}$.

Remark 1.3. Fixing $U$ as above, if $(\mathrm{A}, \xi, \iota) \in \mathbf{P O r d}_{\mathbb{C}, K_{0}}$ corresponds as in Lemma 2.4 to a point $\tau \in U$, then the $d$-tuple

$$
\left(J_{1}, \ldots, J_{d}\right)(\tau)
$$

determines $(\mathrm{A}, \xi, \iota)$ up to isomorphism. That is, on $U, \mathrm{RM}$ isomorphism invariants are isomorphism invariants in the intuitive sense.

Modular polynomials for elliptic curves of level $\ell$ correspond to $\ell$-isogenies of elliptic curves. Hilbert modular polynomials have a level $\mu$, where $\mu$ is a totally positive prime element of $\mathcal{O}_{K_{0}}$, and this corresponds to ' $\mu$-isogenies' between elements of POrd $\mathbf{P}_{\mathbb{C}, K_{0}}$. These isogenies respect the polarisation and the real multiplication; for the formal definition see Definition 2.2.

Let $\mathbb{H}$ be the complex upper half plane. We want to view objects in POrd $\mathbf{C}_{\mathbb{C}, K_{0}}$ as elements of $\mathbb{H}^{g}$, where $g$ is the degree of $K_{0}$ over $\mathbb{Q}$. We will be interested in the action of matrix groups with entries in $K_{0}$ on elements of $\mathbb{H}^{g}$, hence it is much more convenient to work with $K_{0} \otimes \mathbb{C}$ instead of $\mathbb{C}^{g}$. To this end, we fix once for all a $\mathbb{C}$-algebra isomorphism

$$
\mathbb{C}^{g} \longrightarrow K_{0} \otimes \mathbb{C}
$$

and we define $K_{0} \otimes \mathbb{H}$ to be the image of $\mathbb{H}^{g}$ under this isomorphism. Observe that $K_{0} \otimes \mathbb{H}$ does not depend on the choice of isomorphism. 
Definition 1.4. For a totally positive prime element $\mu$ of $\mathcal{O}_{K_{0}}$, and for $\tau, \tau^{\prime} \in$ $K_{0} \otimes \mathbb{H}$, we say that there exists a $\mu$-isogeny

$$
\tau \rightarrow \tau^{\prime}
$$

if there exists a $\mu$-isogeny

$$
(\mathrm{A}, \xi, \iota) \longrightarrow\left(\mathrm{A}^{\prime}, \xi^{\prime}, \iota^{\prime}\right)
$$

where the isomorphism classes of $(\mathrm{A}, \xi, \iota)$ and $\left(\mathrm{A}^{\prime}, \xi^{\prime}, \iota^{\prime}\right) \in \mathbf{P O r d}_{\mathbb{C}, K_{0}}$ correspond as in Lemma 2.4 to the equivalence classes of $\tau$ and $\tau^{\prime}$ in $V$ respectively. (It is well-known that $\tau$ and $\tau^{\prime}$ satisfy

$$
H_{1}(\mathrm{~A}(\mathbb{C}), \mathbb{Z})=\tau \mathcal{O}_{K_{0}}+\mathcal{O}_{K_{0}}^{\vee} \quad \text { and } \quad H_{1}\left(\mathrm{~A}^{\prime}(\mathbb{C}), \mathbb{Z}\right)=\tau^{\prime} \mathcal{O}_{K_{0}}+\mathcal{O}_{K_{0}}^{\vee},
$$

where $H_{1}$ denotes the first singular homology group).

We can now state our main theorem:

Theorem 1.5. For a totally real number field $K_{0}$ of degree $g$ over $\mathbb{Q}$, and a totally positive prime element $\mu$ of $\mathcal{O}_{K_{0}}$, let $\bar{V}$ be the Hilbert modular variety for $K_{0}$ (as defined in Definition 2.13), and fix a choice of RM isomorphism invariant $\left(J_{1}, \ldots, J_{d}\right)$ for $K_{0}$ (as defined in Definition 1.2). Then Algorithm 6.3 outputs a polynomial

$$
G_{\mu}\left(X_{1}, \ldots, X_{d}, Y\right) \in \mathbb{Z}\left[X_{1}, \ldots, X_{d}, Y\right]
$$

that has degree $\operatorname{Norm}_{K_{0} / \mathbb{Q}}(\mu)+1$ in $Y$ and such that the polynomial discriminant $\Delta G_{\mu}\left(J_{1}, \ldots, J_{d}, Y\right)$ is not constant zero on $V$, and outputs polynomials

$$
H_{\mu, i}\left(X_{1}, \ldots, X_{d}, Y, Z_{i}\right) \in \mathbb{Z}\left[X_{1}, \ldots, X_{d}, Y, Z_{i}\right]
$$

that are linear in $Z_{i}$, where $i=2, \ldots d$. Furthermore, for any choice of Zariskiopen subvariety $U$ of $\bar{V}$ such that the map

$$
\left(J_{1}, \ldots, J_{d}\right): U \rightarrow \mathbb{A}_{\mathbb{C}}^{d}
$$

is injective, there exists a codimension $\geq 1$ subset $S$ of $U \cap V$ such that for all

$$
[\tau],\left[\tau^{\prime}\right] \in(U \cap V)-S \cup\left\{x \in(U \cap V): \Delta G_{\mu}\left(J_{1}(x), \ldots, J_{d}(x), Y\right)=0\right\},
$$

there exists a $\mu$-isogeny

$$
\tau \rightarrow \tau^{\prime}
$$

if and only if

$$
G\left(J_{1}(\tau), \ldots, J_{d}(\tau), J_{1}\left(\tau^{\prime}\right)\right)=0
$$

and for $i=2, \ldots, d$,

$$
H_{\mu, i}\left(J_{1}(\tau), \ldots, J_{d}(\tau), J_{1}\left(\tau^{\prime}\right), J_{i}\left(\tau^{\prime}\right)\right)=0 .
$$


Definition 1.6. For a totally positive prime element $\mu \in K_{0}$, we define a set of Hilbert modular polynomials of level $\mu$ to be a set of polynomials

$$
\left\{\begin{array}{c}
G_{\mu}\left(X_{1}, \ldots, X_{d}, Y\right) \in \mathbb{Z}\left[X_{1}, \ldots, X_{d}, Y\right], \\
H_{\mu, i}\left(X_{1}, \ldots, X_{d}, Y, Z_{i}\right) \in \mathbb{Z}\left[X_{1}, \ldots, X_{d}, Y, Z_{i}\right]
\end{array}\right\}_{i=2, \ldots, d}
$$

such that $G_{\mu}\left(X_{1}, \ldots, X_{d}, Y\right)$ and $H_{\mu, i}\left(X_{1}, \ldots, X_{d}, Y, Z_{i}\right)$ satisfy the conclusions of Theorem 1.5.

Remark 1.7. Even though Theorem 1.5 refers only to $\mathbb{C}$, in practise we can use it over finite fields. That is, as the coefficients of the polynomials are integral, we can reduce them modulo a prime $p$, and for $g=2$ the reduced polynomials of level $\mu$ allow us to compute $\mu$-isogenous principally polarised abelian surfaces defined over $\mathbb{F}_{p}$ (see Section 7 for more details).

Remark 1.8. Algorithm 6.3 should be considered primarily as a theoretical algorithm-with the current knowledge the input of the algorithm is only known heuristically and furthermore even when the input is known the algorithm is only practical for a small number of cases.

\section{Preliminaries}

\subsection{Maximal real multiplication}

In this article, we will study principally polarised abelian varieties of dimension $g$ defined over $\mathbb{C}$ that have maximal real multiplication, that is, the real part of the endomorphism ring is a maximal order in a totally real number field of degree $g$ over $\mathbb{Q}$.

Definition 2.1. Let $K_{0}$ be a totally real number field of degree $g$ over $\mathbb{Q}$ with ring of integers $\mathcal{O}_{K_{0}}$, and define $\operatorname{Ord}_{k, g}$ to be the category of ordinary abelian varieties over a field $k$ of dimension $g$. We define the objects of the category $\operatorname{Ord}_{k, K_{0}}$ to be pairs $(\mathrm{A}, \iota)$, where $\mathrm{A} \in \operatorname{Ord}_{k, g}$ and $\iota: \mathcal{O}_{K_{0}} \hookrightarrow \operatorname{End}(\mathrm{A})$ is an embedding. A morphism in $\operatorname{Ord}_{k, K_{0}}$ between two objects $(\mathrm{A}, \iota)$ and $\left(\mathrm{A}^{\prime}, \iota^{\prime}\right)$ is given by a morphism $f: \mathrm{A} \rightarrow \mathrm{A}^{\prime}$ in $\operatorname{Ord}_{k, g}$ such that the following diagram commutes:

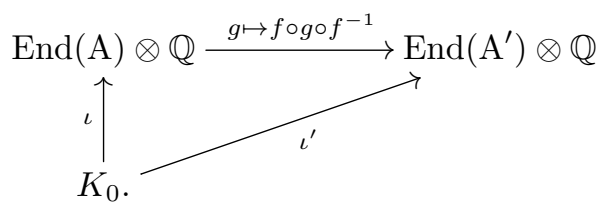

We define the objects of the category $\operatorname{POrd}_{k, K_{0}}$ to be triples $(\mathrm{A}, \xi, \iota)$, where $(\mathrm{A}, \iota) \in \operatorname{Ord}_{k, K_{0}}$ and $\xi: \mathrm{A} \rightarrow \mathrm{A}^{\vee}$ (here $\mathrm{A}^{\vee}$ denotes the dual abelian variety) is a principal polarisation of $\mathrm{A}$, and the image of $\iota$ is stable under the Rosati involution. A morphism in $\operatorname{POrd}_{k, K_{0}}$ between two objects (A, $\left.\xi, \iota\right)$ and $\left(\mathrm{A}^{\prime}, \xi^{\prime}, \iota^{\prime}\right) \in \mathbf{P O r d}_{k, K_{0}}$ is an isomorphism

$$
f:(\mathrm{A}, \iota) \longrightarrow\left(\mathrm{A}^{\prime}, \iota^{\prime}\right)
$$


in $\operatorname{Ord}_{k, K_{0}}$ that makes the following diagram commute:

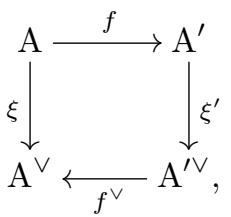

where $\mathrm{A}^{\vee}, \mathrm{A}^{\prime \vee}$, and $f^{\vee}$ are the duals of $\mathrm{A}, \mathrm{A}^{\prime}$, and $f$ respectively.

Definition 2.2. Let $K_{0}$ be a totally real number field with ring of integers $\mathcal{O}_{K_{0}}$ and let $k$ be a field. For

$$
(\mathrm{A}, \xi, \iota),\left(\mathrm{A}^{\prime}, \xi^{\prime}, \iota^{\prime}\right) \in \operatorname{POrd}_{k, K_{0}}
$$

and $\mu \in \mathcal{O}_{K_{0}}$, we define a $\mu$-isogeny $f:(\mathrm{A}, \xi, \iota) \rightarrow\left(\mathrm{A}^{\prime}, \xi^{\prime}, \iota^{\prime}\right)$ to be a morphism $f:(\mathrm{A}, \iota) \rightarrow\left(\mathrm{A}^{\prime}, \iota^{\prime}\right)$ in $\operatorname{Ord}_{k, K_{0}}$ such that the following diagram commutes:

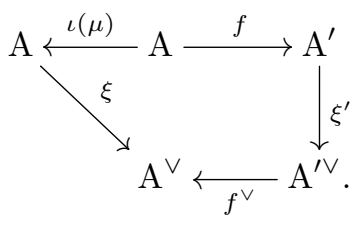

\subsection{Hilbert modular forms}

Following van der Geer [33] we give some preliminaries on Hilbert modular forms; the interested reader should refer to van der Geer for more details.

Definition 2.3. Let $K_{0}$ be a totally real number field of degree $g$ over $\mathbb{Q}$ with ring of integers $\mathcal{O}_{K_{0}}$. Let $\mathcal{N}$ be an invertible $\mathcal{O}_{K_{0}}$-ideal. Then the matrix group $\operatorname{SL}\left(\mathcal{O}_{K_{0}} \oplus \mathcal{N}\right)$ is defined as

$$
\left\{\left(\begin{array}{ll}
a & b \\
c & d
\end{array}\right) \in \mathrm{SL}_{2}\left(K_{0}\right): a, d \in \mathcal{O}_{K_{0}}, b \in \mathcal{N}, c \in \mathcal{N}^{-1}\right\}
$$

Let the group of $2 \times 2$ matrices with entries in $K_{0}$ that have totally positive determinant be denoted by $\mathrm{GL}_{2}\left(K_{0}\right)^{+}$. The group $\mathrm{GL}_{2}\left(K_{0}\right)^{+}$acts on $K_{0} \otimes \mathbb{H}$ as

$$
\left(\begin{array}{ll}
a & b \\
c & d
\end{array}\right) \cdot \tau \mapsto(a \tau+b)(c \tau+d)^{-1}
$$

Lemma 2.4. Let $K_{0}$ be a totally real number field of degree $g$ over $\mathbb{Q}$ with ring of integers $\mathcal{O}_{K_{0}}$, and write $\mathcal{O}_{K_{0}}^{\vee}$ for the trace dual of $\mathcal{O}_{K_{0}}$. Then there is a bijection

$$
\mathrm{SL}\left(\mathcal{O}_{K_{0}} \oplus \mathcal{O}_{K_{0}}^{\vee}\right) \backslash\left(K_{0} \otimes \mathbb{H}\right) \longrightarrow\left\{(\mathrm{A}, \xi, \iota) \in \operatorname{POrd}_{\mathbb{C}, K_{0}}\right\}_{/ \cong}
$$


where the image of $\tau \in \operatorname{SL}\left(\mathcal{O}_{K_{0}} \oplus \mathcal{O}_{K_{0}}^{\vee}\right) \backslash\left(K_{0} \otimes \mathbb{H}\right)$ is

$$
\mathrm{A}=\left(K_{0} \otimes \mathbb{C}\right) /\left(\tau \mathcal{O}_{K_{0}}+\mathcal{O}_{K_{0}}^{\vee}\right)
$$

with the natural embedding $\iota$ and the polarisation induced by the Riemann form $E:\left(K_{0} \otimes \mathbb{C}\right) \times\left(K_{0} \otimes \mathbb{C}\right) \longrightarrow \mathbb{R}$ given by

$$
E\left(\tau u_{1}+u_{2}, \tau v_{1}+v_{2}\right)=\operatorname{tr}_{\left(K_{0} \otimes \mathbb{R}\right) / \mathbb{R}}\left(u_{1} v_{2}-u_{2} v_{1}\right)
$$

for $u_{1}, u_{2}, v_{1}, v_{2} \in K_{0} \otimes \mathbb{R}$.

Proof. See [33, Chapter IX, Section 1].

Definition 2.5. Let $\kappa$ be an integer, and let $\tau$ be in $K_{0} \otimes \mathbb{H}$. Then the weight function $w_{\kappa}$ is defined by

$$
\begin{array}{ccc}
\mathrm{GL}_{2}\left(K_{0}\right)^{+} \times\left(K_{0} \otimes \mathbb{H}\right) & \longrightarrow & \mathbb{C} \\
(M, \tau) & \mapsto & \left(\mathrm{N}_{K_{0} / \mathbb{Q}}(\operatorname{det}(M))^{-\frac{1}{2}} \mathrm{~N}_{\left(K_{0} \otimes \mathbb{C}\right) / \mathbb{C}}(c \tau+d)\right)^{\kappa},
\end{array}
$$

where we choose the positive square root.

Definition 2.6. Let $\mathrm{GL}_{2}\left(K_{0}\right)^{+}$and $K_{0} \otimes \mathbb{H}$ be as above. Let $M$ be any matrix in $\mathrm{GL}_{2}\left(K_{0}\right)^{+}$, and let $f: K_{0} \otimes \mathbb{H} \rightarrow \mathbb{C}$ be a holomorphic map. Then we define $\left.f\right|_{[M]_{\kappa}}$ by

$$
\begin{array}{cccc}
\left.f\right|_{[M]_{\kappa}}: \quad K_{0} \otimes \mathbb{H} & \rightarrow & \mathbb{C} \\
\tau & \mapsto & w_{\kappa}(M, \tau)^{-1} f(M \tau) .
\end{array}
$$

It is straightforward to check that for $M, N \in \mathrm{GL}_{2}\left(K_{0}\right)^{+}$, we have

$$
\left.\left(\left.f\right|_{[M]_{\kappa}}\right)\right|_{[N]_{\kappa}}=\left.f\right|_{[M N]_{\kappa}} .
$$

Definition 2.7. Let $\mathrm{GL}_{2}\left(K_{0}\right)^{+}$and $K_{0} \otimes \mathbb{H}$ be as above, and assume that $g>1$. Let $\Gamma$ be a congruence subgroup of $\mathrm{GL}_{2}\left(K_{0}\right)^{+}$. We say that $f: K_{0} \otimes \mathbb{H} \rightarrow \mathbb{C}$ is a Hilbert modular form of weight $\kappa$ for $\Gamma$ if and only if it is holomorphic and for all $M \in \Gamma$ and $\tau \in K_{0} \otimes \mathbb{H}$, we have

$$
\left.f\right|_{[M]_{\kappa}}(\tau)=f(\tau) .
$$

From this point on, if $f$ is a Hilbert modular form of weight $\kappa$, then for $M \in$ $\mathrm{GL}_{2}\left(K_{0}\right)^{+}$we will write $\left.f\right|_{M}$ for $\left.f\right|_{[M]_{\kappa}}$.

Remark 2.8. For $g=1$, we also impose holomorphicity at the cusps (see e.g. [35. p4]).

Definition 2.9. With notation as in Definition 2.7 if $\varphi=f / g$ is the quotient of Hilbert modular forms for $\Gamma$ of equal weight, then we say that $\varphi$ is a Hilbert modular function for $\Gamma$. 
Definition 2.10. Let $\mathcal{O}_{K_{0}}^{\vee}$ be the trace dual of $\mathcal{O}_{K_{0}}$. We define $\mathcal{M}_{K_{0}, \kappa}$ to be the $\mathbb{C}$-vector space of Hilbert modular forms for $\operatorname{SL}\left(\mathcal{O}_{K_{0}} \oplus \mathcal{O}_{K_{0}}^{\vee}\right)$ of weight $\kappa$, and we define

$$
\mathcal{M}_{K_{0}}=\bigoplus_{\kappa} \mathcal{M}_{K_{0}, \kappa}
$$

to be the graded $\mathbb{C}$-algebra of all Hilbert modular forms for $\mathrm{SL}\left(\mathcal{O}_{K_{0}} \oplus \mathcal{O}_{K_{0}}^{\vee}\right)$. For $f \in \mathcal{M}_{K_{0}}$, let coeffs $(f)$ be the set of coefficients of the $q$-expansion of $f$ around the cusp at infinity. For a ring $R$, we define

$$
\mathcal{M}_{K_{0}, \kappa}(R)=\left\{f \in \mathcal{M}_{K_{0}, \kappa}: \operatorname{coeffs}(f) \subseteq R\right\},
$$

and

$$
\mathcal{M}_{K_{0}}(R)=\left\{f \in \mathcal{M}_{K_{0}}: \operatorname{coeffs}(f) \subseteq R\right\} .
$$

Definition 2.11. Suppose that $g=2$. Then for $f \in \mathcal{M}_{K_{0}, k}$, if for every $\left(\tau_{1}, \tau_{2}\right) \in K_{0} \otimes \mathbb{H}=\mathbb{H}^{2}$ we have

$$
f\left(\tau_{1}, \tau_{2}\right)=f\left(\tau_{2}, \tau_{1}\right),
$$

we say that $f$ is symmetric.

Theorem 2.12. (Baily-Borel Theorem)

Let $\mathcal{M}_{K_{0}}$ be the graded ring of Hilbert modular forms for $\mathrm{SL}\left(\mathcal{O}_{K_{0}} \oplus \mathcal{O}_{K_{0}}^{\vee}\right)$. Then the normal complex analytic space of $\operatorname{Proj}\left(\mathcal{M}_{K_{0}}\right)$ is a compactification of

$$
V=\operatorname{SL}\left(\mathcal{O}_{K_{0}} \oplus \mathcal{O}_{K_{0}}^{\vee}\right) \backslash\left(K_{0} \otimes \mathbb{H}\right) \text {. }
$$

Proof. See [33, Theorem II.7.1].

Definition 2.13. We define the Hilbert modular variety $\bar{V}$ to be the normal complex analytic space of $\operatorname{Proj}\left(\mathcal{M}_{K_{0}}\right)$. We will also refer to this as the Baily-Borel compactification of $V$.

Proposition 2.14. (Rapoport)

$\mathcal{M}_{K_{0}, \kappa}(\mathbb{Z})$ is a finitely generated $\mathbb{Z}$-module.

Proof. See [28, Proposition 6.6].

Lemma 2.15. (Rapoport)

$$
\mathcal{M}_{K_{0}}(\mathbb{Z}) \otimes_{\mathbb{Z}} \mathbb{C}=\mathcal{M}_{K_{0}} .
$$

Proof. See the proof of [28, Lemme 6.12].

Proposition 2.16. Let $K_{0}$ be a quadratic number field of discriminant 5, 8, 13 or 17 . Then $\mathcal{M}_{K_{0}}(\mathbb{Q})$ is a finitely generated $\mathbb{Q}$-algebra, and the $q$-expansions of a choice of generators are known.

Proof. For discriminant 5 see [26] or [21], for discriminant 8 see [25], and for discriminants 13 and 17 see [21].

Assumption 2.17. In everything that follows, we will assume that $\mathcal{M}_{K_{0}}(\mathbb{Q})$ is a finitely generated $\mathbb{Q}$-algebra. 


\section{Defining RM isomorphism invariants}

As before, let $K_{0}$ be a totally real number field of degree $g$ over $\mathbb{Q}$, and let $\bar{V}$ be the Hilbert modular variety for $\operatorname{SL}\left(\mathcal{O}_{K_{0}} \oplus \mathcal{O}_{K_{0}}^{\vee}\right)$, as defined in Definition 2.13. The aim of this section is to prove Proposition 3.1.

For completeness, we recall here the definition of RM isomorphism invariants from above.

Definition 1.2, A d-tuple of $\mathbb{Q}$-linearly independant, non-constant Hilbert modular functions

$$
\left(J_{1}, \ldots, J_{d}\right) \in \mathbb{Q}\left(\mathcal{M}_{K_{0}}(\mathbb{Z})\right)^{\times d}
$$

such that

$$
\mathbb{C}(\bar{V})=\mathbb{C}\left(J_{1}, \ldots, J_{d}\right)
$$

is a choice of RM isomorphism invariants for $K_{0}$.

Proposition 3.1. Write $\mathbb{Q}\left(\mathcal{M}_{K_{0}}(\mathbb{Z})\right)$ for the $\mathbb{Q}$-algebra of quotients of Hilbert modular forms in $\mathcal{M}_{K_{0}}(\mathbb{Z})$ of equal weight; assume as in Assumption 2.17 that this is finitely generated as a $\mathbb{Q}$-algebra. There exists $d \in \mathbb{Z}$ and a choice

$$
J_{1}, \ldots, J_{d} \in \mathbb{Q}\left(\mathcal{M}_{K_{0}}(\mathbb{Z})\right)
$$

of RM isomorphism invariants for $K_{0}$. Furthermore, for such $J_{1}, \ldots, J_{d}$, there exists a Zariski-open affine subvariety $U$ of $\bar{V}$ such that the map

$$
\left(J_{1}, \ldots, J_{d}\right): U \longrightarrow \mathbb{A}_{\mathbb{C}}^{d}
$$

is a well-defined injective morphism.

Proof. Write $\mathbb{C}\left(\mathcal{M}_{K_{0}}\right)$ for the field of quotients of elements of $\mathcal{M}_{K_{0}}$ of equal weight. By definition of $\bar{V}$ (see Definition 2.13), we have that $\mathbb{C}(\bar{V})=\mathbb{C}\left(\mathcal{M}_{K_{0}}\right)$, and by Lemma 2.15, we know that

$$
\mathbb{Q}\left(\mathcal{M}_{K_{0}}(\mathbb{Z})\right) \otimes_{\mathbb{Q}} \mathbb{C}=\mathbb{C}\left(\mathcal{M}_{K_{0}}\right) .
$$

So let $J_{1}, \ldots, J_{d}$ be generators of the $\mathbb{Q}$-algebra $\mathbb{Q}\left(\mathcal{M}_{K_{0}}(\mathbb{Z})\right)$, so that

$$
\mathbb{C}\left(J_{1}, \ldots, J_{d}\right)=\mathbb{C}(\bar{V}),
$$

and write $W$ for the image of $\left(J_{1}, \ldots, J_{d}\right)$ in $\mathbb{A}_{\mathbb{C}}^{d}$. Then by [15, Corollary I.4.5], there are non-empty Zariski-open subsets $U \subseteq \bar{V}$ and $U^{\prime} \subseteq W$ such that $U$ is isomorphic to $U^{\prime}$.

Example 3.2. If $g=1$, so that $K_{0}=\mathbb{Q}$, then we have that

$$
\mathrm{SL}_{2}\left(\mathcal{O}_{K_{0}} \oplus \mathcal{O}_{K_{0}}^{\vee}\right) \backslash K_{0} \otimes \mathbb{H}=\mathrm{SL}_{2}(\mathbb{Z}) \backslash \mathbb{H} \text {. }
$$

The $j$-invariant for elliptic curves defines an isomorphism

$$
j: \mathrm{SL}_{2}(\mathbb{Z}) \backslash \mathbb{H} \longrightarrow \mathbb{A}_{\mathbb{C}}^{1}
$$

Hence setting

$$
V=\mathrm{SL}_{2}(\mathbb{Z}) \backslash \mathbb{H}, \quad \bar{V}=\mathbb{P}_{\mathbb{C}}^{1}, \quad U=V, \text { and } \quad J_{1}=j
$$

gives us $\mathbb{C}(\bar{V})=\mathbb{C}\left(J_{1}\right)$ and an injective morphism $J_{1}: U \rightarrow \mathbb{A}_{\mathbb{C}}^{1}$. 


\section{Computing $\mu$-isogenous elements in the moduli space}

As before, in what follows, $K_{0}$ is a totally real number field of degree $g$ over $\mathbb{Q}$ with ring of integers $\mathcal{O}_{K_{0}}$. The aim of this section is to prove Proposition 4.5. This Proposition concerns the polynomials

$$
\Phi_{\mu}(Y) \in \mathcal{M}_{K_{0}}(\mathbb{Z})[Y]
$$

and

$$
\Psi_{\mu, i}\left(Y, Z_{i}\right) \in \mathcal{M}_{K_{0}}(\mathbb{Z})\left[Y, Z_{i}\right]
$$

(defined below) from which, given a 'sufficiently generic' element $\tau \in K_{0} \otimes \mathbb{H}$ we can read off the $\tau^{\prime} \in K_{0} \otimes \mathbb{H}$ that are $\mu$-isogenous to $\tau$ (recall the definition of $\mu$-isogeny in this context from Definition 1.4. These polynomials are an important stepping stone to computing the set of Hilbert modular polynomials of Theorem 1.5.

From this point on, we fix RM isomorphism invariants $\left(J_{1}, \ldots, J_{d}\right) \in \mathbb{Q}\left(\mathcal{M}_{K_{0}}(\mathbb{Z})\right)^{\times d}$, and a non-empty Zariski-open subvariety $U$ of the Hilbert modular variety $\bar{V}$ such that

$$
\left(J_{1}, \ldots, J_{d}\right): U \longrightarrow \mathbb{A}_{\mathbb{C}}^{d}
$$

defines an injective morphism.

For $i=1, \ldots, d$, we choose $f_{i}$ and $g_{i}$ to be elements of $\mathcal{M}_{K_{0}}(\mathbb{Z})$ of weight $k_{i}$ such that

$$
J_{i}=f_{i} / g_{i} .
$$

Definition 4.1. Let $\operatorname{SL}\left(\mathcal{O}_{K_{0}} \oplus \mathcal{O}_{K_{0}}^{\vee}\right)$ be as in Definition 2.3 and let $\mu$ be a totally positive prime element of $\mathcal{O}_{K_{0}}$. Define

$$
\Gamma^{0}(\mu):=\left\{\left(\begin{array}{cc}
a & b \\
c & d
\end{array}\right) \in \operatorname{SL}\left(\mathcal{O}_{K_{0}} \oplus \mathcal{O}_{K_{0}}^{\vee}\right): b \in \mu \mathcal{O}_{K_{0}}^{\vee}\right\}
$$

For any $x \in K_{0}$ define

$$
\underline{x}:=\left(\begin{array}{cc}
x & 0 \\
0 & 1
\end{array}\right) .
$$

Lemma 4.2. Given a Hilbert modular form $f \in \mathcal{M}_{K_{0}}(\mathbb{Z})$, for every $N \in$ $\operatorname{SL}\left(\mathcal{O}_{K_{0}} \oplus \mathcal{O}_{K_{0}}^{\vee}\right)$, the function $\left.f\right|_{\mu^{-1} N}$ depends only on the class of $N$ in

$$
\Gamma^{0}(\mu) \backslash \mathrm{SL}\left(\mathcal{O}_{K_{0}} \oplus \mathcal{O}_{K_{0}}^{\vee}\right)
$$

Proof. Let

$$
M:=\left(\begin{array}{ll}
a & b \\
c & d
\end{array}\right) \in \Gamma^{0}(\mu)
$$

and let

$$
M^{\prime}:=\left(\begin{array}{cc}
a & \mu^{-1} b \\
\mu c & d
\end{array}\right)
$$


so that $\mu^{-1} M=M^{\prime} \mu^{-1}$. As $b \in \mu \mathcal{O}_{K_{0}}^{\vee}$ and $\operatorname{det}\left(M^{\prime}\right)=1$ we have that $M^{\prime} \in$ $\operatorname{SL}\left(\mathcal{O}_{K_{0}} \oplus \mathcal{O}_{K_{0}}^{\vee}\right)$. In particular

$$
\left.f\right|_{\underline{\mu^{-1} M N}}=\left.f\right|_{M^{\prime} \underline{\mu^{-1} N}}=\left.\left(\left.f\right|_{M^{\prime}}\right)\right|_{\underline{\mu^{-1} N}}=\left.f\right|_{\underline{\mu^{-1} N}} .
$$

Definition 4.3. Denote by $\mathcal{C}$ a choice of coset representatives for the quotient of groups

$$
\Gamma^{0}(\mu) \backslash \mathrm{SL}\left(\mathcal{O}_{K_{0}} \oplus \mathcal{O}_{K_{0}}^{\vee}\right) .
$$

We then further define

$$
\Phi_{\mu}(Y):=\prod_{M \in \mathcal{C}}\left(\left.g_{1}\right|_{\underline{\mu^{-1}}} M-\left.f_{1}\right|_{\underline{\mu^{-1}} M}\right)
$$

and for each $i=2, \ldots, d$,

$$
\begin{aligned}
\Psi_{\mu, i}\left(Y, Z_{i}\right):=\sum_{M \in \mathcal{C}}\{ & \left(\left.g_{i}\right|_{\underline{\mu^{-1}} M} Z_{i}-\left.f_{i}\right|_{\underline{\mu^{-1}} M}\right) \\
& \left.\cdot \prod_{\substack{M^{\prime} \in \mathcal{C} \\
M^{\prime} \neq M}}\left(\left.g_{1}\right|_{\underline{\mu^{-1}}} M^{\prime} Y-\left.f_{1}\right|_{\underline{\mu^{-1}}} M^{\prime}\right)\right\} .
\end{aligned}
$$

Note that the definitions of $\Phi_{\mu}(Y)$ and $\Psi_{\mu, i}\left(Y, Z_{i}\right)$ do not depend on the choice of coset representatives for $\Gamma^{0}(\mu) \backslash \mathrm{SL}\left(\mathcal{O}_{K_{0}} \oplus \mathcal{O}_{K_{0}}^{\vee}\right)$.

Lemma 4.4. We have that

$$
\Phi_{\mu}(Y) \in \mathcal{M}_{K_{0}}(\mathbb{Z})[Y] \quad \text { and } \quad \Psi_{\mu, i}\left(Y, Z_{i}\right) \in \mathcal{M}_{K_{0}}(\mathbb{Z})\left[Y, Z_{i}\right] .
$$

Proof. Recall that for $M \in \mathcal{C}$ and $N \in \operatorname{SL}\left(\mathcal{O}_{K_{0}} \oplus \mathcal{O}_{K_{0}}^{\vee}\right)$, for every $f \in \mathcal{M}_{K_{0}}$, we have that

$$
\left.\left(\left.f\right|_{\underline{\mu^{-1} M}}\right)\right|_{N}(\tau)=\left.f\right|_{\underline{\mu^{-1} M N}}(\tau) .
$$

In particular, acting by $\left.\right|_{N}$ on the coefficients of $\Phi_{\mu}(Y)$ (and $\Psi_{\mu, i}\left(Y, Z_{i}\right)$ ) just permutes the factors (or terms) of the defining product (or sum), leaving $\Phi_{\mu}(Y)$ (and $\Psi_{\mu, i}\left(Y, Z_{i}\right)$ ) unchanged, hence the coefficients are modular forms for $\mathrm{SL}\left(\mathcal{O}_{K_{0}} \oplus \mathcal{O}_{K_{0}}^{\vee}\right)$.

As $\Phi_{\mu}$ is a univariate polynomial with coefficients that are modular forms for $\operatorname{SL}\left(\mathcal{O}_{K_{0}} \oplus \mathcal{O}_{K_{0}}^{\vee}\right)$ of equal weight, the discriminant $\Delta \Phi_{\mu}$ is also a modular form for $\operatorname{SL}\left(\mathcal{O}_{K_{0}} \oplus \mathcal{O}_{K_{0}}^{\vee}\right)$. In particular, whether or not $\left(\Delta \Phi_{\mu}\right)(\tau)=0$ depends only on the class of $\tau$ in $V$.

Proposition 4.5. Fix notation as in Definition 4.3 and recall from Definition 1.4 the definition of a $\mu$-isogeny $\tau \rightarrow \tau^{\prime}$ for $\tau, \tau^{\prime} \in K_{0} \otimes \mathbb{H}$. For any $\tau, \tau^{\prime} \in K_{0} \otimes \mathbb{H}$ such that the classes $[\tau]$ and $\left[\tau^{\prime}\right]$ of $\tau$ and $\tau^{\prime}$ in $\bar{V}$ are in

$$
(U \cap V)-\left\{x \in(U \cap V):\left(\Delta \Phi_{\mu}\right)(x)=0\right\},
$$


there exists a $\mu$-isogeny $\tau \rightarrow \tau^{\prime}$ if and only if for every $i=2, \ldots, d$, evaluating $\Phi_{\mu}(Y)$ and $\Psi_{\mu, i}\left(Y, Z_{i}\right)$ at $\left(Y, Z_{2}, \ldots, Z_{d}\right)=\left(J_{1}\left(\left[\tau^{\prime}\right]\right), \ldots, J_{d}\left(\left[\tau^{\prime}\right]\right)\right)$, and then evaluating the resulting modular forms at $\tau$, gives

$$
\left(\Phi_{\mu}\left(J_{1}\left(\left[\tau^{\prime}\right]\right)\right)\right)(\tau)=0 \quad \text { and } \quad\left(\Psi_{\mu, i}\left(J_{1}\left(\left[\tau^{\prime}\right]\right), J_{i}\left(\left[\tau^{\prime}\right]\right)\right)\right)(\tau)=0 .
$$

We will prove Proposition 4.5 at the end of this section, by using the following lemma and a representation of $\mu$-isogenies up to isomorphism.

Lemma 4.6. If $\mu$ is a totally positive prime element of $\mathcal{O}_{K_{0}}$ then the set $\Gamma^{0}(\mu) \backslash \mathrm{SL}\left(\mathcal{O}_{K_{0}} \oplus \mathcal{O}_{K_{0}}^{\vee}\right)$ has $\operatorname{Norm}_{K_{0} / \mathbb{Q}}(\mu)+1$ elements.

Proof. Define

$$
k:=\max \left\{n \in \mathbb{Z}:\left(\mathcal{O}_{K_{0}}^{\vee}\right)^{-1} \subseteq \mu^{n} \mathcal{O}_{K_{0}}\right\} .
$$

There is a bijection of sets

$$
\begin{aligned}
& \Gamma^{0}(\mu) \backslash \mathrm{SL}\left(\mathcal{O}_{K_{0}} \oplus \mathcal{O}_{K_{0}}^{\vee}\right) \longleftrightarrow\left(\underline{\mu^{k}} \Gamma^{0}(\mu) \underline{\mu^{-k}}\right) \backslash\left(\underline{\mu^{k}} \operatorname{SL}\left(\mathcal{O}_{K_{0}} \oplus \mathcal{O}_{K_{0}}^{\vee} \underline{\mu^{-k}}\right)\right. \\
& M \quad \mapsto \quad-\quad \underline{\mu^{k}} M \mu^{-k} .
\end{aligned}
$$

We claim that

$$
\left\{\left(\begin{array}{ll}
1 & a \\
0 & 1
\end{array}\right): a \in \mathcal{O}_{K_{0}} / \mu \mathcal{O}_{K_{0}}\right\} \cup\left\{\left(\begin{array}{cc}
0 & 1 \\
-1 & 0
\end{array}\right)\right\}
$$

is in bijection with $\left(\underline{\mu^{k}} \Gamma^{0}(\mu) \underline{\mu^{-k}}\right) \backslash\left(\underline{\mu^{k}} \operatorname{SL}\left(\mathcal{O}_{K_{0}} \oplus \mathcal{O}_{K_{0}}^{\vee}\right) \underline{\mu^{-k}}\right)$. Let

$$
\left(\begin{array}{cc}
a & b \\
c & d
\end{array}\right) \in \underline{\mu^{k}} \operatorname{SL}\left(\mathcal{O}_{K_{0}} \oplus \mathcal{O}_{K_{0}}^{\vee}\right) \underline{\mu^{-k}} .
$$

Then $a, d \in \mathcal{O}_{K_{0}}, b \in \mu^{k} \mathcal{O}_{K_{0}}^{\vee}$ and $c \in \mu^{-k}\left(\mathcal{O}_{K_{0}}^{\vee}\right)^{-1}$. For every fractional ideal $\mathcal{N}$ of $\mathcal{O}_{K_{0}}$, denote the localisation of $\mathcal{N}$ at the prime ideal $\mu \mathcal{O}_{K_{0}}$ by $\mathcal{N}_{(\mu)}$. As $\mathcal{O}_{K_{0}}^{\vee}$ is an invertible $\mathcal{O}_{K_{0}}$-ideal and $\left(\mathcal{O}_{K_{0}}\right)_{(\mu)}$ is a local ring, we have that $\left(\mathcal{O}_{K_{0}}^{\vee}\right)_{(\mu)}$ is a principal fractional $\left(\mathcal{O}_{K_{0}}\right)_{(\mu)}$-ideal. Define $\alpha \in K_{0}$ such that $\left(\mu^{k} \mathcal{O}_{K_{0}}^{\vee}\right)_{(\mu)}=\alpha\left(\mathcal{O}_{K_{0}}\right)_{(\mu)}$. Then the following defines a group homomorphism:

$$
\begin{aligned}
r: \underline{\mu^{k} \operatorname{SL}\left(\mathcal{O}_{K_{0}} \oplus \mathcal{O}_{K_{0}}^{\vee}\right) \underline{\mu^{-k}}} & \rightarrow \quad \operatorname{SL}_{2}\left(\mathcal{O}_{K_{0}} / \mu \mathcal{O}_{K_{0}}\right) \\
\left(\begin{array}{ll}
a & b \\
c & d
\end{array}\right) & \mapsto\left(\begin{array}{cc}
a+\mu \mathcal{O}_{K_{0}} & \alpha^{-1} b+\mu \mathcal{O}_{K_{0}} \\
\alpha c+\mu \mathcal{O}_{K_{0}} & d+\mu \mathcal{O}_{K_{0}}
\end{array}\right) .
\end{aligned}
$$

Now $\mathcal{O}_{K_{0}} / \mu \mathcal{O}_{K_{0}}$ is a field as $\mu \mathcal{O}_{K_{0}}$ is prime, and $\mathrm{SL}_{2}\left(\mathcal{O}_{K_{0}} / \mu \mathcal{O}_{K_{0}}\right)$ acts on $\mathbb{P}^{1}\left(\mathcal{O}_{K_{0}} / \mu \mathcal{O}_{K_{0}}\right)$ as

$$
\left(\begin{array}{ll}
a & b \\
c & d
\end{array}\right) \cdot(x: y) \mapsto(a x+b y: c x+d y) .
$$

The stabilizer of $(0: 1)$ is

$$
\left\{\left(\begin{array}{cc}
a & 0 \\
c & d
\end{array}\right) \in \mathrm{SL}_{2}\left(\mathcal{O}_{K_{0}} / \mu \mathcal{O}_{K_{0}}\right)\right\}
$$

the pull-back of which under $r$ is $\underline{\mu^{k}} \Gamma^{0}(\mu) \underline{\mu^{-k}}$, so the bijection follows from the orbit-stabilizer theorem. 
Definition 4.7. We say $\mu$-isogenies

$$
f:\left(\mathrm{A}, \xi_{\mathrm{A}}, \iota_{\mathrm{A}}\right) \rightarrow\left(\mathrm{B}, \xi_{\mathrm{B}}, \iota_{\mathrm{B}}\right) \text { and } g:\left(\mathrm{A}, \xi_{\mathrm{A}}, \iota_{\mathrm{A}}\right) \rightarrow\left(\mathrm{B}^{\prime}, \xi_{\mathrm{B}^{\prime}}, \iota_{\mathrm{B}^{\prime}}\right)
$$

are isomorphic if there exists a 1-isogeny $\varphi:\left(\mathrm{B}, \xi_{\mathrm{B}}, \iota_{\mathrm{B}}\right) \rightarrow\left(\mathrm{B}^{\prime}, \xi_{\mathrm{B}^{\prime}}, \iota_{\mathrm{B}^{\prime}}\right)$ such that the diagram

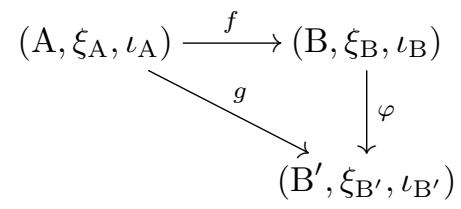

commutes.

Definition 4.8. For every $\left(\begin{array}{ll}a & b \\ c & d\end{array}\right)=M \in \mathrm{GL}_{2}\left(K_{0}\right)^{+}$and for every $\tau \in$ $K_{0} \otimes \mathbb{H}$, we define $\varphi_{M, \tau}$ to be the element of $\operatorname{Hom}_{\operatorname{Ord}_{\mathfrak{C}, K_{0}}}(\tau, M \tau) \otimes \mathbb{Q}$ that is multiplication by $(c \tau+d)^{-1}$ on $K_{0} \otimes \mathbb{C}$.

Note that

$$
\varphi_{M, N \tau} \circ \varphi_{N, \tau}=\varphi_{M N, \tau}
$$

and

$$
\varphi_{M, \tau}^{-1}=\varphi_{M^{-1}, M \tau} .
$$

Lemma 4.9. We have that $\varphi_{M, \tau}$ is an isomorphism in $\operatorname{POrd}_{\mathbb{C}, K_{0}}$ if and only if $M \in \operatorname{SL}\left(\mathcal{O}_{K_{0}} \oplus \mathcal{O}_{K_{0}}^{\vee}\right)$.

Proof. Write $M=\left(\begin{array}{ll}a & b \\ c & d\end{array}\right)$ and for any $\tau^{\prime} \in K_{0} \otimes \mathbb{H}$ let $E_{\tau^{\prime}}$ be the Riemann form

$$
E_{\tau^{\prime}}\left(u_{1} \tau+u_{2}, v_{1} \tau^{\prime}+v_{2}\right)=\operatorname{tr}_{K_{0} / \mathbb{Q}}\left(u_{1} v_{2}-u_{2} v_{1}\right) .
$$

We get a commutative diagram of unpolarised abelian varieties, where the dashed arrows are automorphisms of $K_{0} \otimes \mathbb{C}$ that may or may not induce actual maps of abelian varieties:

$$
\begin{aligned}
& \left(K_{0} \otimes \mathbb{C}\right) /\left(\tau \mathcal{O}_{K_{0}}+\mathcal{O}_{K_{0}}^{\vee}\right) \stackrel{\varphi_{M, \tau}:=(c \tau+d)^{-1}}{-}-\stackrel{-}{\rightarrow}\left(K_{0} \otimes \mathbb{C}\right) /\left(M \tau \mathcal{O}_{K_{0}}+\mathcal{O}_{K_{0}}^{\vee}\right) \\
& \ldots \quad:=\mathrm{id}_{\left(K_{0} \otimes \mathrm{C}\right)} \quad \downarrow \quad c \tau+d \\
& \left.\left(K_{0} \otimes \mathbb{C}\right) /(\overrightarrow{(a \tau}+b){\stackrel{\mathcal{O}}{K_{0}}}+(c \tau+d) \mathcal{O}_{K_{0}}^{\vee}\right) .
\end{aligned}
$$

Now $f$, and hence $\varphi_{M, \tau}$ defines an isomorphism on lattices if and only if $M \in$ $\operatorname{GL}\left(\mathcal{O}_{K_{0}}+\mathcal{O}_{K_{0}}^{\vee}\right)$. Suppose now that $M \in \operatorname{GL}\left(\mathcal{O}_{K_{0}}+\mathcal{O}_{K_{0}}^{\vee}\right)$. It remains to show that $\operatorname{det}(M)=1$ if and only if $\varphi_{M, \tau}$ is an isomorphism in $\operatorname{POrd}_{\mathbb{C}, K_{0}}$, that is, if

$$
E_{\tau}(\alpha, \beta)=E_{M \tau}\left(\varphi_{M, \tau}(\alpha), \varphi_{M, \tau}(\beta)\right) .
$$


Write $E_{\tau}=\operatorname{tr}_{K_{0} / \mathbb{Q}} \circ S_{\tau}$ and $E_{M \tau}=\operatorname{tr}_{K_{0} / \mathbb{Q}} \circ S_{M \tau}$. The matrices of $S_{\tau}$ and $\varphi_{M, \tau}^{*} S_{M \tau}$ with respect to the $\left(K_{0} \otimes \mathbb{R}\right)$-basis $\{\tau, 1\}$ of $K_{0} \otimes \mathbb{C}$ are

$$
\left(\begin{array}{cc}
0 & 1 \\
-1 & 0
\end{array}\right)
$$

and

$$
M\left(\begin{array}{cc}
0 & 1 \\
-1 & 0
\end{array}\right) M^{t}
$$

respectively, so $S_{\tau}=\varphi_{M, \tau}^{*} S_{M \tau}$ if and only if $\operatorname{det}(M)=1$ and the result follows.

Lemma 4.10. Fix a totally positive prime element $\mu \in K_{0}$. Then for any $\tau \in K_{0} \otimes \mathbb{H}$, there is a map

$$
\begin{array}{ccc}
i: \Gamma^{0}(\mu) \backslash \operatorname{SL}\left(\mathcal{O}_{K_{0}} \oplus \mathcal{O}_{K_{0}}^{\vee}\right) & \longrightarrow\{\mu \text {-isogenies from } \tau\}_{/ \cong} \\
M & \mapsto \varphi_{\underline{\mu^{-1}, M \tau}} \circ \varphi_{M, \tau},
\end{array}
$$

and $i$ defines a bijection of sets.

Proof. Observe that $\mathrm{id}_{K_{0} \otimes \mathbb{C}}$ defines a $\mu$-isogeny

$$
\begin{aligned}
\varphi_{\underline{\mu^{-1}, \tau}} & :\left(\left(K_{0} \otimes \mathbb{C}\right) /\left(\tau \mathcal{O}_{K_{0}}+\mathcal{O}_{K_{0}}^{\vee}\right), \xi, \iota\right) \\
& \longrightarrow\left(\left(K_{0} \otimes \mathbb{C}\right) /\left(\underline{\left.\left.\mu^{-1} \tau \mathcal{O}_{K_{0}}+\mathcal{O}_{K_{0}}^{\vee}\right), \mu \xi, \iota\right),}\right.\right.
\end{aligned}
$$

where $\xi$ is a principal polarisation of $\left(K_{0} \otimes \mathbb{C}\right) /\left(\tau \mathcal{O}_{K_{0}}+\mathcal{O}_{K_{0}}^{\vee}\right)$, which in other words is a $\mu$-isogeny $\tau \rightarrow \mu^{-1} \tau$.

We claim that $i$ is a well-defined injection of sets. Let $M, N \in \operatorname{SL}\left(\mathcal{O}_{K_{0}} \oplus \mathcal{O}_{K_{0}}^{\vee}\right)$ and suppose that $\varphi_{\mu^{-1}, M \tau} \circ \varphi_{M, \tau}$ and $\varphi_{\mu^{-1}, N \tau} \circ \varphi_{N, \tau}$ are isomorphic as $\mu$-isogenies. That is, there exists an isomorphism

$$
\psi: \underline{\mu^{-1}} M \tau \rightarrow \underline{\mu^{-1}} N \tau
$$

such that

$$
\psi \circ \varphi_{\underline{\mu^{-1}}, M \tau} \circ \varphi_{M, \tau}=\varphi_{\underline{\mu^{-1}}, N \tau} \circ \varphi_{N, \tau},
$$

hence by (3) and (4)

$$
\psi=\varphi_{\underline{\mu^{-1}}} N M^{-1} \underline{\underline{\mu}, \underline{\mu^{-1}}} M \tau .
$$

By Lemma 4.9 as $\psi$ is an isomorphism, we have that

$$
\underline{\mu^{-1}} N M^{-1} \underline{\mu} \in \operatorname{SL}\left(\mathcal{O}_{K_{0}} \oplus \mathcal{O}_{K_{0}}^{\vee}\right) .
$$

Define $X=N M^{-1}$ and $T=\underline{\mu^{-1} N M^{-1}} \underline{\mu}$. As $T$ and $X \in \operatorname{SL}\left(\mathcal{O}_{K_{0}} \oplus \mathcal{O}_{K_{0}}^{\vee}\right)$, we get further that $X \in \Gamma^{0}(\mu)$-that is, the matrices $M$ and $N$ are in the same coset of $\Gamma^{0}(\mu) \backslash \operatorname{SL}\left(\mathcal{O}_{K_{0}} \oplus \mathcal{O}_{K_{0}}^{\vee}\right)$. Conversely, suppose that $N M^{-1} \in \Gamma^{0}(\mu)$. Then

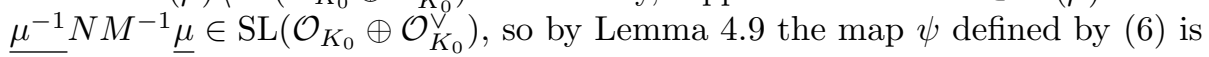
an isomorphism. Hence $i$ is a well-defined injection of sets. 
To show that $i$ is in fact a bijection we proceed by counting. By Lemma 4.6 the set $\mathcal{C}$ (c.f. Definition 4.3 has $\operatorname{Norm}_{K_{0} / \mathbb{Q}}(\mu)+1$ elements, so we just need to show that there are at most $\operatorname{Norm}_{K_{0} / \mathbb{Q}}(\mu)+1$ non-isomorphic $\mu$-isogenies from any given $\tau \in K_{0} \otimes \mathbb{H}$. If $f:\left(\mathrm{A}, \xi_{\mathrm{A}}, \iota_{\mathrm{A}}\right) \rightarrow\left(\mathrm{B}, \xi_{\mathrm{B}}, \iota_{\mathrm{B}}\right)$ is a $\mu$-isogeny, then

$$
\operatorname{ker}(f) \subseteq \operatorname{ker}(\mu) \cong\left(\mathcal{O}_{K_{0}} / \mu \mathcal{O}_{K_{0}}\right)^{\times 2} .
$$

Also, as for every $\alpha \in \mathcal{O}_{K_{0}}$ the following diagram commutes:

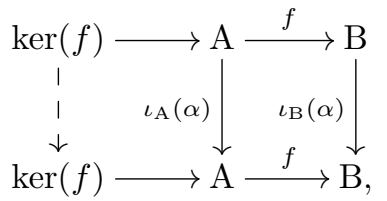

the kernel of $f$ is an $\mathcal{O}_{K_{0}}$-module, and hence an $\mathcal{O}_{K_{0}} / \mu \mathcal{O}_{K_{0}}$ sub-vector space of $\left(\mathcal{O}_{K_{0}} / \mu \mathcal{O}_{K_{0}}\right)^{\times 2}$. Then, as $\operatorname{deg}(f)=\operatorname{Norm}_{K_{0} / \mathbb{Q}}(\mu)$, there are at most

$\operatorname{Norm}_{K_{0} / \mathbb{Q}}(\mu)+1$ distinct kernels of $\mu$-isogenies from any given $\tau$ (or equivalently any given $\left.(\mathrm{A}, \xi, \iota) \in \mathbf{P O r d}_{\mathbb{C}, K_{0}}\right)$. Therefore it remains to show that there do not exist non-isomorphic $\mu$-isogenies

$$
f:\left(\mathrm{A}, \xi_{\mathrm{A}}, \iota_{\mathrm{A}}\right) \rightarrow\left(\mathrm{B}, \xi_{\mathrm{B}}, \iota_{\mathrm{B}}\right)
$$

and

$$
f^{\prime}:\left(\mathrm{A}, \xi_{\mathrm{A}}, \iota_{\mathrm{A}}\right) \rightarrow\left(\mathrm{B}^{\prime}, \xi_{\mathrm{B}^{\prime}}, \iota_{\mathrm{B}^{\prime}}\right)
$$

with the same kernel. By the universal property of quotient maps there exists an isomorphism $\alpha$ (of unpolarised abelian varieties) such that the following diagram commutes:

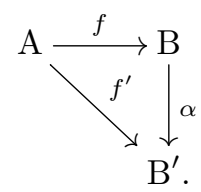

We claim that $\alpha$ is a 1 -isogeny. Consider the following diagram:

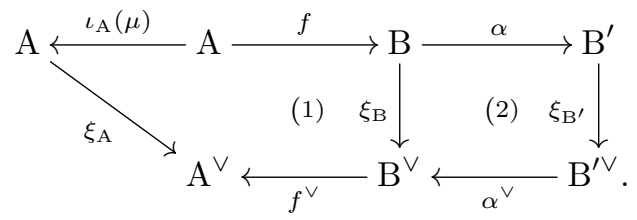

Diagram (1) commutes as $f$ is a $\mu$-isogeny and the diagram formed by the outside arrows commutes as $f^{\prime}$ is a $\mu$-isogeny, hence diagram (2) commutes. Similarly, consider the following diagram:

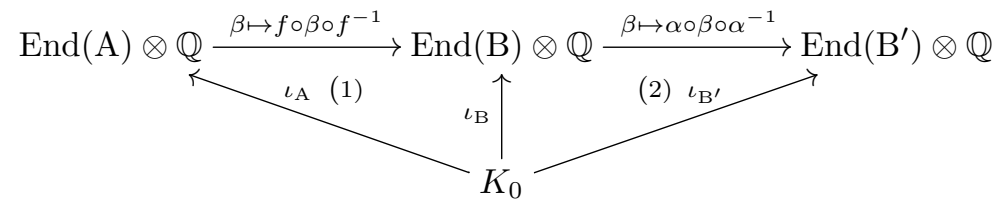


Diagram (1) commutes as $f$ is a $\mu$-isogeny and the diagram formed by the outside arrows commutes as $f^{\prime}$ is a $\mu$-isogeny and

$$
f^{\prime} \circ \beta \circ\left(f^{\prime}\right)^{-1}=(\alpha \circ f) \circ \beta \circ(\alpha \circ f)^{-1}=\alpha \circ\left(f \circ \beta \circ f^{-1}\right) \circ \alpha^{-1} .
$$

Hence (2) commutes. Now: $\alpha$ defines an isomorphism of abelian varieties, diagram (2) of Equation (7) commutes, and diagram (2) of Equation (8) commutes. So by definition $\alpha:\left(\mathrm{B}, \xi_{\mathrm{B}}, \iota_{\mathrm{B}}\right) \rightarrow\left(\mathrm{B}^{\prime}, \xi_{\mathrm{B}^{\prime}}, \iota_{\mathrm{B}^{\prime}}\right)$ is a 1-isogeny. Hence $f$ and $f^{\prime}$ are isomorphic as $\mu$-isogenies.

Proof of Proposition 4.5. Suppose first that there exists a $\mu$-isogeny $\tau \rightarrow \tau^{\prime}$. Then by Lemma 4.10, there exists $N \in \mathcal{C}=\Gamma^{0}(\mu) \backslash \operatorname{SL}\left(\mathcal{O}_{K_{0}} \oplus \mathcal{O}_{K_{0}}^{\vee}\right)$ such that this $\mu$-isogeny is isomorphic to a $\mu$-isogeny $\tau \rightarrow \mu^{-1} N \tau$, so we can identify $\tau^{\prime}$ with $\underline{\mu^{-1} N \tau}$. Plugging this into the definitions of $\overline{\Phi_{\mu}}(Y)$ and $\Psi_{\mu, i}\left(Y, Z_{i}\right)$, we get

$$
\Phi_{\mu}\left(J_{1}\left(\underline{\mu^{-1}} N \tau\right)\right)=0
$$

and

$$
\Psi_{\mu, i}\left(J_{1}\left(\underline{\mu^{-1}} N \tau\right), J_{i}\left(\underline{\mu^{-1}} N \tau\right)\right)=0 .
$$

Suppose now that $\left(Y_{0}, Z_{2,0}, \ldots, Z_{d, 0}\right)$ is a common root of $\Phi_{\mu}(Y)$ and $\Psi_{\mu, i}\left(Y, Z_{i}\right)$. One can see directly from the definition of $\Phi_{\mu}$ and $\Psi_{\mu, i}$ that under the discriminant condition, the set of common roots of 4.3 is exactly the set

$$
\left\{\left(J_{1}\left(\underline{\mu^{-1}} M \tau\right), \ldots, J_{d}\left(\underline{\mu^{-1}} M \tau\right)\right): M \in \mathcal{C}\right\} .
$$

Therefore, there exists $N \in \mathcal{C}$ such that

$$
\left(Y_{0}, Z_{2,0}, \ldots, Z_{d, 0}\right)=\left(J_{1}\left(\underline{\mu^{-1}} N \tau\right), \ldots, J_{d}\left(\underline{\mu^{-1}} N \tau\right)\right),
$$

and by Lemma 4.10 there exists a $\mu$-isogeny

$$
\tau \rightarrow \underline{\mu^{-1}} N \tau \text {. }
$$

\section{Computing the RM isomorphism invariants for a given genus 2 curve}

In Definition 1.2, we defined RM isomorphism invariants for elements of POrd ${\mathbb{C}, K_{0}}_{0}$. Restrict now to the dimension 2 case. It is however not immediately clear how to compute these given the equation of a genus 2 curve. We have a computational advantage in genus 2 , which is that there already exist IgusaClebsch invariants to determine a curve up to isomorphism.

Definition 5.1. For a curve $C$ of genus 2 over a field $k$ with $\operatorname{char}(k) \neq 2$, there exists a hyperelliptic model $y^{2}=f(x)$ of $C$, where $f$ is a separable polynomial of degree 6. Fix such a model, denote by $c$ the leading coefficient of $f$, fix an ordering $x_{1}, \ldots, x_{6}$ of the roots of $f$ in its splitting field, and denote by $(i j)$ the 
difference $x_{i}-x_{j}$. For $\operatorname{char}(k) \neq 2,3,5$, we define the Igusa-Clebsch invariants of $C$ to be

$$
\begin{aligned}
I_{2} & =c^{2} \sum(12)^{2}(34)^{2}(56)^{2}, \\
I_{4} & =c^{4} \sum(12)^{2}(23)^{2}(31)^{2}(45)^{2}(56)^{2}(64)^{2}, \\
I_{6} & =c^{6} \sum(12)^{2}(23)^{2}(31)^{2}(45)^{2}(56)^{2}(64)^{2}(14)^{2}(25)^{2}(36)^{2}, \\
I_{10} & =c^{10} \prod(12)^{2},
\end{aligned}
$$

where each sum and product runs over the distinct expressions obtained by applying a permutation to the index set $\{1, \ldots, 6\}$.

These invariants are integral whenever $f$ is integral. The Igusa-Clebsch invariants are 'invariants for the Siegel moduli space'. Before making this more precise, we recall some facts about the Siegel moduli space.

Definition 5.2. We define

$$
\operatorname{Sym}_{2}(\mathbb{C})=\left\{\left(\begin{array}{cc}
\tau_{1} & \tau_{2} \\
\tau_{2} & \tau_{3}
\end{array}\right) \in \operatorname{Mat}_{2 \times 2}(\mathbb{C})\right\},
$$

and for $\tau \in \operatorname{Sym}_{2}(\mathbb{C})$, we write $\operatorname{Im}(\tau)>0$ for ' $\operatorname{Im}(\tau)$ is positive definite'.

Definition 5.3. The Siegel upper half space is defined to be

$$
\mathbb{H}_{2}=\left\{\tau=\left(\begin{array}{ll}
\tau_{1} & \tau_{2} \\
\tau_{2} & \tau_{3}
\end{array}\right) \in \operatorname{Sym}_{2}(\mathbb{C}): \operatorname{Im}(\tau)>0\right\},
$$

and the symplectic group

$$
\mathrm{Sp}_{2}(\mathbb{Z})=\left\{\gamma \in \mathrm{GL}_{4}(\mathbb{Z}): \gamma\left(\begin{array}{cc}
0 & I_{2} \\
-I_{2} & 0
\end{array}\right) \gamma^{\mathrm{tr}}=\left(\begin{array}{cc}
0 & I_{2} \\
-I_{2} & 0
\end{array}\right)\right\}
$$

acts on $\mathbb{H}_{2}$ via

$$
\left(\begin{array}{cc}
A & B \\
C & D
\end{array}\right) \cdot \tau=(A \tau+B)(C \tau+D)^{-1} .
$$

The field of rational functions of the coarse moduli space for hyperelliptic curves of genus 2 can be generated by three Siegel modular functions, as shown by Igusa in [16. Following the notation in the Echidna database [17, we choose as generators three Siegel modular functions

$$
i_{1}, i_{2}, i_{3}: \mathrm{Sp}_{2}(\mathbb{Z}) \backslash \mathbb{H}_{2} \longrightarrow \mathbb{C}
$$

such that, if $C$ is a curve of genus 2 , and $[\tau] \in \mathrm{Sp}_{2}(\mathbb{Z}) \backslash \mathbb{H}_{2}$ is the point in the moduli space corresponding to $C$, then

$$
\begin{aligned}
& i_{1}(\tau)=\left(I_{4} I_{6} / I_{10}\right)(C), \\
& i_{2}(\tau)=\left(I_{2}^{3} I_{4} / I_{10}\right)(C), \\
& i_{3}(\tau)=\left(I_{2}^{2} I_{6} / I_{10}\right)(C) .
\end{aligned}
$$


Now, for a totally real quadratic number field $K_{0}$, the forgetful functor

$$
\begin{aligned}
& \operatorname{POrd}_{\mathbb{C}, K_{0}} \longrightarrow \operatorname{POrd}_{\mathbb{C}, 2} \\
& (\mathrm{~A}, \xi, \iota) \quad \mapsto \quad(\mathrm{A}, \xi)
\end{aligned}
$$

induces a map

$$
\phi: \operatorname{SL}\left(\mathcal{O}_{K_{0}} \oplus \mathcal{O}_{K_{0}}^{\vee}\right) \backslash K_{0} \otimes \mathbb{H} \rightarrow \operatorname{Sp}_{2}(\mathbb{Z}) \backslash \mathbb{H}_{2},
$$

which is generically $2-1$. We will refer to this as the modular map. The image of this map is called the Humbert surface for $K_{0}$, and is denoted as $\mathcal{H}_{K_{0}}$. That is, the modular map $\phi$ induces a degree 2 map

$$
\phi: \mathcal{M}_{K_{0}} \longrightarrow \mathcal{H}_{K_{0}}
$$

In particular, as there exist 2 algebraically independent Siegel modular functions $f_{1}$ and $f_{2}$ in

$$
\mathbb{C}\left(\mathcal{H}_{K_{0}}\right) \subseteq \mathbb{C}\left(i_{1}, i_{2}, i_{3}\right),
$$

we get 2 algebraically independent Hilbert modular functions

$$
J_{1}=\phi^{*} f_{1} \quad \text { and } \quad J_{2}=\phi^{*} f_{2}
$$

in $\mathbb{C}\left(\mathcal{M}_{K_{0}}\right)$. Also, by construction, we get that $J_{1}$ and $J_{2}$ are symmetric, that is, that if $\sigma$ is the generator of $\operatorname{Gal}\left(K_{0} / \mathbb{Q}\right)$, then for all $\tau \in K_{0} \otimes \mathbb{H}$, we have that

$$
J_{1}(\sigma(\tau))=J_{1}(\tau) \quad \text { and } \quad J_{2}(\sigma(\tau))=J_{2}(\tau) .
$$

Formally extend $J_{1}, J_{2}$ to a full set of RM isomorphism invariants $J_{1}, \ldots, J_{d}$ in the sense of Definition 1.2 Recall from Definition 1.2 that $J_{1}, \ldots, J_{d}$ are $\mathbb{Q}$-linearly independent and generate the $\mathbb{Q}$-algebra $\mathbb{Q}\left(\mathcal{M}_{K_{0}}(\mathbb{Z})\right)$ and from Lemma 2.15 and Definition 2.13 that

$$
\mathbb{Q}\left(\mathcal{M}_{K_{0}}(\mathbb{Z})\right) \otimes_{\mathbb{Q}} \mathbb{C}=\mathbb{C}\left(\mathcal{M}_{K_{0}}\right)=\mathbb{C}\left(J_{1}, \ldots, J_{d}\right)=\mathbb{C}(\bar{V}) .
$$

In particular $\mathbb{C}(\bar{V})$ is a finite separable field extension of $\mathbb{C}\left(J_{1}, J_{2}\right)$ and hence can in fact be generated by one element; choose such an element and denote it by $J_{3}$. Write $m(X) \in \mathbb{C}\left(J_{1}, J_{2}\right)[X]$ for the minimal polynomial of $J_{3}$; then $m(X)$ is the pullback along $\phi$ of a polynomial in $\mathbb{C}\left(i_{1}, i_{2}, i_{3}\right)[X]$. The subtlety of how to choose the root of $m(X)$ in practice is addressed in Algorithm 7.5, Step 2.

Note that Assumption 2.17 is not in fact an assumption in genus 2, and recall that we have explicit generators for the cases given in Proposition 2.16.

Example 5.4. Gundlach [14 and Müller 26] computed formulae for a choice of isomorphism invariants $J_{1}, J_{2}$, and $J_{3}$ for $K_{0}=\mathbb{Q}(\sqrt{5})$, and gave the functions from which $J_{1}, J_{2}$, and $J_{3}^{2}$ (here $m(X)$ is quadratic and without a linear term) 
are pulled back along $\phi$ :

$$
\begin{aligned}
J_{1}= & \phi^{*}\left(\frac{2^{-6} 3^{-3} i_{1}^{2} i_{2}^{2}+2^{-3} 3^{2} i_{1} i_{2}^{2}-2^{-4} 3^{-3} i_{1} i_{3}^{3}+2^{-5} 3^{2} i_{2} i_{3}^{2}}{i_{1}^{2} i_{2}^{2}+2^{2} 3^{5} i_{1} i_{2}^{2}}\right), \\
J_{2}= & \phi^{*}\left(\frac{2^{9} i_{1}^{3} i_{2}^{2}+2^{11} 3^{5} i_{1}^{2} i_{2}^{2}}{i_{1}^{2} i_{2}^{2}+2^{2} i_{1} i_{3}^{3}-2 \cdot 3^{5} i_{2} i_{3}^{2}}\right), \\
J_{3}^{2}= & 5^{5}-2^{-1} 5^{3} J_{1} J_{2}+2^{-4} J_{2}+2^{-1} 3^{2} 5^{2} J_{2}^{2} J_{1}^{3}-2^{-3} J_{1}^{2} J_{2}^{2} \\
& -2 \cdot 3^{3} J_{2}^{3} J_{1}^{5}+2^{-4} J_{2}^{3} J_{1}^{4} .
\end{aligned}
$$

Remark 5.5. For each choice of $K_{0}$, we have to recalculate RM isomorphism invariants $J_{1}, J_{2}$, and $J_{3}$. In [19, Theorem 2.2], Lauter, Naehrig, and Yang give a method to compute the pullbacks of the theta constants along the modular map, from which it should be possible to calculate a choice of Siegel modular functions $f_{1}$ and $f_{2}$ as in $(12)$, but the minimal polynomial of $J_{3}$ over $\mathbb{Q}\left(J_{1}, J_{2}\right)$ is not known in general.

Recall from Lemma 2.15 that $\mathbb{C}(\bar{V})=\mathbb{Q}\left(\mathcal{M}_{K_{0}}(\mathbb{Z})\right) \otimes \mathbb{C}$, so that in particular a choice of $\mathbb{Q}$-algebra generators $J_{1}, \ldots, J_{d}$ for $\mathbb{Q}\left(\mathcal{M}_{K_{0}}(\mathbb{Z})\right)$ is also a choice of $\mathbb{C}$-algebra generators for $\mathbb{C}(\bar{V})$. In the cases for which a complete set of generators is known, namely $K_{0}$ of discriminant $5,8,13$, and 17 , we can choose $\mathrm{RM}$ isomorphism invariants $J_{1}, J_{2}, J_{3} \in \mathbb{Q}\left(\mathcal{M}_{K_{0}}(\mathbb{Z})\right)^{\times 3}$ for which $J_{1}$ and $J_{2}$ are symmetric Hilbert modular functions (as above) and $J_{3}^{2} \in \mathbb{Q}\left(J_{1}, J_{2}\right)$. For simplicity, when working in genus 2 , we restrict to this case in all that follows.

\section{The algorithm in dimension $g$}

Let $\Phi_{\mu}(Y)$ and $\Psi_{\mu, i}\left(Y, Z_{i}\right)$ be as given in Definition 4.3 . Given any choice of coset representatives for $\Gamma^{0}(\mu) \backslash \mathrm{SL}\left(\mathcal{O}_{K_{0}} \oplus \mathcal{O}_{K_{0}}^{\vee}\right)$ it is possible, just by writing out the definitions, to write out explicit formulae for the $q$-expansions of the coefficients of $\Phi_{\mu}(Y)$ and $\Psi_{\mu, i}\left(Y, Z_{i}\right)$. We now outline how to use such Fourier expansions to compute a set of Hilbert modular polynomials.

Suppose that we have a choice $\gamma_{1}, \ldots, \gamma_{s}$ of $\mathbb{Q}$-algebra generators of $\mathcal{M}_{K_{0}}(\mathbb{Q})$, and that for each $\gamma_{i}$ we know its weight $\kappa_{i}$ and can compute its Fourier expansion. Then for each coefficient $f \in \mathcal{M}_{K_{0}}(\mathbb{Z})$ of $\Phi_{\mu}(Y)$ or $\Psi_{\mu, i}\left(Y, Z_{i}\right)$ it is just linear algebra to determine integers $h_{1}, \ldots, h_{s}$ and rational numbers $b_{\underline{h}}$, where $\underline{h}=$ $\left(h_{1}, \ldots, h_{s}\right)$, such that

$$
f=\sum_{\left\{\underline{h} \in\left(\mathbb{Z}_{\geq 0}\right)^{s}: \sum_{j=1}^{s} h_{j} \kappa_{j}=k\right\}} b_{\underline{h}} \prod_{j=1}^{s} \gamma_{j}^{h_{j}},
$$

where $k$ is the weight of $f$ : Suppose that $\left\{\delta_{1}, \ldots, \delta_{g}\right\}$ defines an integral basis of $\mathcal{O}_{K_{0}}^{\vee}$, define $q_{n}=e^{2 \pi i \operatorname{tr}\left(\delta_{n} \tau\right)}$, and for $\left(t_{1}, \ldots, t_{g}\right)=\underline{t} \in \mathbb{Z}^{g}$, define

$$
\underline{q}^{\underline{t}}=\prod_{n=1}^{g} q_{n}^{t_{n}}
$$


Then for each $\underline{h}$ appearing in the summand range of (16), there exist rational numbers $\beta_{\underline{h}}(\underline{t})$ such that

$$
\prod_{j=1}^{s} \gamma_{j}^{h_{j}}=\sum_{\underline{t} \in \mathbb{Z}^{g}} \beta_{\underline{h}}(\underline{t}) \underline{q^{\underline{t}}} .
$$

Of course, there also exist rational numbers $\alpha(\underline{t})$ such that

$$
f=\sum_{\underline{t} \in \mathbb{Z}^{g}} \alpha(\underline{t}) \underline{q}^{\underline{t}} .
$$

Then, writing $\left\{\underline{t}_{1}, \ldots\right\}=\left\{\underline{t} \in \mathbb{Z}^{g}\right\}$ and

$$
\left\{\underline{h}_{1}, \ldots, \underline{h}_{r}\right\}=\left\{\underline{h} \in\left(\mathbb{Z}_{\geq 0}\right)^{s}: \sum_{j=1}^{s} h_{j} \kappa_{j}=k\right\}
$$

we can rewrite equation 16 as

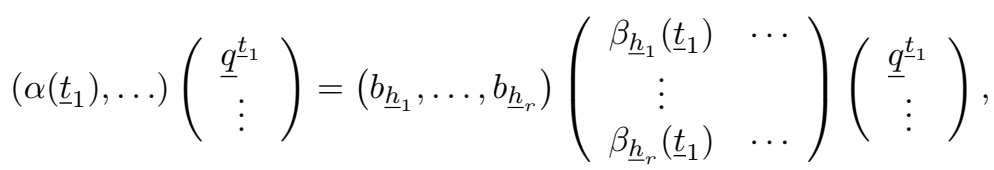

which we can now solve just with linear algebra over $\mathbb{Q}$. At some cut-off point $\underline{t}_{N}$ the linear system will be completely determined: one should choose the precision $P$ up to which to input the coefficients of the Fourier expansions $\gamma_{1}, \ldots, \gamma_{s}$ into the computer in such a way that this system has a unique solution for every coefficient $f \in \mathcal{M}_{K_{0}}(\mathbb{Z})$ of $\Phi_{\mu}(Y)$ or $\Psi_{\mu, i}\left(Y, Z_{i}\right)$. For details on how to do this in a somewhat optimised way for the case that is fully implemented (i.e. $g=2$ and $K_{0}=\mathbb{Q}(\sqrt{5})$ ), see the MAGMA code at www.martindale. info/research.

Assumption 6.1. From now on, we assume that we are in case in which we have a choice $\gamma_{1}, \ldots, \gamma_{s} \in \mathcal{M}_{K_{0}}(\mathbb{Z})$ of $\mathbb{Q}$-algebra generators of $\mathcal{M}_{K_{0}}(\mathbb{Q})$ for which:

1. We know the weights $\kappa_{1}, \ldots, \kappa_{s}$ of $\gamma_{1}, \ldots, \gamma_{s}$ respectively.

2. We know the coefficients of the Fourier expansions of $\gamma_{1}, \ldots, \gamma_{s}$ up to the required precision $P$.

Recall from Proposition 2.16 that this assumption holds (at least) for quadratic $K_{0}$ of discriminant $5,8,13$, or 17 .

To deduce the set of Hilbert modular polynomials $G_{\mu}$ and $H_{\mu, i}$ (c.f. Definition 1.6 from $\Phi_{\mu}$ and $\Psi_{\mu, i}$, we first have to scale $\Phi_{\mu}$ and $\Psi_{\mu, i}$ so that the coefficients are in $\mathbb{Q}\left(\mathcal{M}_{K_{0}}(\mathbb{Z})\right)$. To do this, we construct a ring homomorphism

$$
\mathcal{M}_{K_{0}}(\mathbb{Z}) \longrightarrow \mathbb{Q}\left(\mathcal{M}_{K_{0}}(\mathbb{Z})\right) \text {. }
$$

To this end, we define

$$
\lambda=\operatorname{gcd}\left(\left\{\sum_{i=1}^{s} c_{i} \kappa_{i}:\left(c_{1}, \ldots, c_{s}\right) \in \mathbb{Z}^{\times s}\right\}\right)=\operatorname{gcd}\left(\left\{\kappa_{1}, \ldots, \kappa_{s}\right\}\right),
$$


and choose $w_{1}$ and $w_{2}$ such that $\mathcal{M}_{K_{0}, w_{1}}(\mathbb{Z}), \mathcal{M}_{K_{0}, w_{2}}(\mathbb{Z}) \neq \emptyset$ and $\lambda=w_{1}-w_{2}$.

Then choose

$$
\varphi \in \mathcal{M}_{K_{0}, w_{2}}(\mathbb{Z}) \text { and } \quad \psi \in \mathcal{M}_{K_{0}, w_{1}}(\mathbb{Z}),
$$

and define

$$
\varphi_{i}=\varphi^{\kappa_{i} / \lambda} \quad \text { and } \quad \psi_{i}=\psi^{\kappa_{i} / \lambda} .
$$

This defines a map

$$
\begin{array}{ccc}
\mathcal{M}_{K_{0}, \kappa_{i}}(\mathbb{Z}) & \longrightarrow & \mathbb{Q}\left(\mathcal{M}_{K_{0}}(\mathbb{Z})\right) \\
\gamma_{i} & \mapsto & \frac{\varphi_{i}}{\psi_{i}} \gamma_{i}
\end{array}
$$

which extends $\mathbb{Z}$-linearly to a map

$$
\rho: \mathcal{M}_{K_{0}}(\mathbb{Z}) \longrightarrow \mathbb{Q}\left(\mathcal{M}_{K_{0}}(\mathbb{Z})\right),
$$

which is in fact a ring homomorphism. In Algorithm 6.3, we will assume that the representations of $\rho\left(\gamma_{1}\right), \ldots, \rho\left(\gamma_{s}\right)$ as rational functions in $J_{1}, \ldots, J_{d}$ are known.

Example 6.2. Müller 26] defined four elements $\left(\gamma_{1}, \gamma_{2}, \gamma_{3}, \gamma_{4}\right)=\left(g_{2}, s_{5}, g_{6}, s_{15}\right)$ of $\mathcal{M}_{\mathbb{Q}(\sqrt{5})}(\mathbb{Z})$ of weights $2,5,6$, and 15 respectively that generate $\mathcal{M}_{\mathbb{Q}(\sqrt{5})}(\mathbb{Q})$ as a $\mathbb{Q}$-algebra and defined modular functions

$$
\left(J_{1}, J_{2}, J_{3}\right)=\left(\frac{g_{2}^{5}}{s_{5}^{2}}, \frac{s_{6}}{g_{2}^{3}}, \frac{s_{5}^{3}}{s_{15}}\right),
$$

such that $\mathbb{Q}\left(\mathcal{M}_{K_{0}}(\mathbb{Z})\right)=\mathbb{Q}\left(J_{1}, J_{2}, J_{3}\right)$. In this case, we get that $\lambda=1$, we choose $w_{1}=5$ and $w_{2}=4$, and we choose $\varphi=g_{2}^{2}$ and $\psi=s_{5}$. Then

$$
\begin{aligned}
& \gamma_{1}=g_{2} \mapsto \frac{g_{2}^{5}}{s_{5}^{2}}=J_{1} \\
& \gamma_{2}=s_{5} \mapsto \frac{g_{2}^{10}}{s_{5}^{4}}=\left(\frac{g_{2}^{5}}{s_{5}^{2}}\right)^{2}=J_{1}^{2} \\
& \gamma_{3}=s_{6} \mapsto \frac{g_{2}^{12} s_{6}}{s_{5}^{6}}=\left(\frac{g_{2}^{5}}{s_{5}^{2}}\right)^{3} \frac{s_{6}}{g_{2}^{3}}=J_{1}^{3} J_{2} \\
& \gamma_{4}=s_{15} \mapsto \frac{g_{2}^{30} s_{15}}{s_{5}^{15}}=\left(\frac{g_{2}^{5}}{s_{5}^{2}}\right)^{6} \frac{s_{15}}{s_{5}^{3}}=J_{1}^{6} J_{3}^{-1} .
\end{aligned}
$$

The choice given in Equation (20) is the choice in the implementation of Algorithm 6.3 that can be found at www.martindale.info/research

Algorithm 6.3 is a theoretical algorithm to compute a set of Hilbert modular polynomials in the sense of Definition 1.6. Note that in practise the inputs to this algorithm are difficult to compute - more research is needed before we can compute the $\gamma_{i}$ and related quantities for dimensions higher than two. This is beyond the scope of this work so we leave this as an open question. 


\section{Algorithm 6.3.}

INPUT: A totally real number field $K_{0}$ of degree $g$ over $\mathbb{Q}$, the $q$-expansions of generators $\gamma_{1}, \ldots, \gamma_{s}$ of the $\mathbb{Q}$-algebra $\mathcal{M}_{K_{0}}(\mathbb{Q})$ up to the precision $P$ explained above, the images of $\gamma_{1}, \ldots, \gamma_{s}$ under $\rho$ as rational functions of $J_{1}, \ldots, J_{d}$, and a totally positive element $\mu \in \mathcal{O}_{K_{0}}$ that generates a prime ideal.

OUTPUT: Polynomials

$$
\begin{aligned}
G_{\mu}\left(X_{1}, \ldots, X_{d}, Y\right) & \in \mathbb{Z}\left[X_{1}, \ldots, X_{d}, Y\right] \\
H_{\mu, i}\left(X_{1}, \ldots, X_{d}, Y, Z_{i}\right) & \in \mathbb{Z}\left[X_{1}, \ldots, X_{d}, Y, Z_{i}\right],
\end{aligned}
$$

for $i=2, \ldots, d$, satisfying the conclusions of Theorem 1.5 .

1. Compute the $q$-expansions of the coefficients of $\Phi_{\mu}$ and $\Psi_{\mu, i}$ up to precision $P$. For more details in genus 2, see Lemma 7.3.

2. As in 16) or 17), write each coefficient of $\Phi_{\mu}$ and $\Psi_{\mu, i}$ as elements of $\mathbb{Z}\left[\gamma_{1}, \ldots, \gamma_{s}\right]$ using linear algebra on the Fourier expansions.

3. Define $\tilde{\rho}$ to be $i \circ \rho$, where $i$ is the ring homomorphism defined by

$$
\begin{array}{ccc}
i: \mathbb{Q}\left(J_{1}, \ldots, J_{d}\right) & \longrightarrow & \mathbb{Q}\left(X_{1}, \ldots, X_{d}\right) \\
J_{i} & \mapsto & X_{i} .
\end{array}
$$

Define

$$
G_{\mu}\left(X_{1}, \ldots, X_{d}, Y\right) \in \mathbb{Z}\left[X_{1}, \ldots, X_{d}, Y\right]
$$

to be the numerator of $\tilde{\rho}\left(\Phi_{\mu}(Y)\right)$ and

$$
H_{\mu, i}\left(X_{1}, \ldots, X_{d}, Y, Z_{i}\right) \in \mathbb{Z}\left[X_{1}, \ldots, X_{d}, Y, Z_{i}\right]
$$

to be the numerator of $\tilde{\rho}\left(\Psi_{\mu, i}\left(Y, Z_{i}\right)\right)$. (To apply $\tilde{\rho}$ to a polynomial with coefficients in $\mathcal{M}_{K_{0}}(\mathbb{Q})$, just apply $\tilde{\rho}$ to the coefficients).

We have implemented a more optimised version of this in MAGMA for $K_{0}=\mathbb{Q}(\sqrt{5})$ and $K_{0}=\mathbb{Q}(\sqrt{2})$, see Section 7. That the output of Algorithm 6.3 is correct was in the statement of Theorem 1.5, which we now prove:

Proof of Theorem 1.5. Define $\tilde{D}_{1}\left(X_{1}, \ldots, X_{d}\right)$ and $\tilde{D}_{i}\left(X_{1}, \ldots, X_{d}\right) \in \mathbb{Z}\left[X_{1}, \ldots, X_{d}\right]$ to be the denominators of $\tilde{\rho}\left(\Phi_{\mu}(Y)\right)$ and $\tilde{\rho}\left(\Psi_{\mu, i}\left(Y, Z_{i}\right)\right)$ respectively, and define

$$
D=\prod_{i=1}^{d} \tilde{D}_{i}\left(J_{1}, \ldots, J_{d}\right) \in \mathbb{Q}\left(\mathcal{M}_{K_{0}}(\mathbb{Z})\right) .
$$

Then $D$ is a Hilbert modular function, so extends to a quotient of holomorphic functions on the compact algebraic variety $\bar{V}$. A holomorphic function on a compact algebraic variety has finitely many poles and if it has zeroes then by Chow's Theorem the zero-locus defines a closed algebraic subvariety. In particular, as $D$ is not identically zero and $\bar{V}$ is irreducible (see [1, Theorem $10.4]$ ), the zero-locus of the numerator (resp. denominator) is either empty or defines an algebraic subvariety of $\bar{V}$ of codimension at least one. Let $S$ be the 
set of all $[\tau] \in U \cap V$ for which $\tau$ defines a zero or pole of $D$; then $S$ is a subset of $U \cap V$ of codimension at least one.

Suppose now that $[\tau] \notin S$. It is immediate from Proposition 4.5 that the roots of $\left(\Phi_{\mu}(Y)\right)(\tau)$ are given by the first isomorphism invariant $J_{1}\left(\tau^{\prime}\right)$ of all the $\tau^{\prime} \in K_{0} \otimes \mathbb{H}$ that are $\mu$-isogeneous to $\tau$, up to isomorphism. If all the $J_{1}\left(\tau^{\prime}\right)$ are distinct then it also follows from Proposition 4.5 that the unique root of $\left(\Psi_{\mu, i}\left(J_{1}\left(\tau^{\prime}\right), Z_{i}\right)\right)(\tau)$ is $J_{i}\left(\tau^{\prime}\right)$. If they are not distinct then $\left(\Delta \Phi_{\mu}\right)(\tau)=0$, so as $[\tau] \notin S$, we have that $\Delta G_{\mu}\left(J_{1}(\tau), \ldots, J_{d}(\tau), Y\right)=0$. Hence, for every

$$
[\tau],\left[\tau^{\prime}\right] \in(U \cap V)-S \cup\left\{x \in(U \cap V): \Delta G_{\mu}\left(J_{1}(x), \ldots, J_{d}(x), Y\right)=0\right\},
$$

there exists a $\mu$-isogeny $\tau \rightarrow \tau^{\prime}$ if and only if $\left(\Phi_{\mu}\left(J_{1}\left(\tau^{\prime}\right)\right)(\tau)=0\right.$ and for $i=2, \ldots, d$, we have that $\left(\Psi_{\mu, i}\left(J_{1}\left(\tau^{\prime}\right), J_{i}\left(\tau^{\prime}\right)\right)(\tau)=0\right.$. But for every

$$
[\tau],\left[\tau^{\prime}\right] \in(U \cap V)-S \cup\left\{x \in(U \cap V): \Delta G_{\mu}\left(J_{1}(x), \ldots, J_{d}(x), Y\right)=0\right\},
$$

we have that $\left(\Phi_{\mu}\left(J_{1}\left(\tau^{\prime}\right)\right)(\tau)=0\right.$ if and only if

$$
G_{\mu}\left(J_{1}(\tau), \ldots, J_{d}(\tau), J_{1}\left(\tau^{\prime}\right)=0\right.
$$

and, for $i=2, \ldots, d$, we have that $\left(\Psi_{\mu, i}\left(J_{1}\left(\tau^{\prime}\right), J_{i}\left(\tau^{\prime}\right)\right)(\tau)=0\right.$ if and only if

$$
H_{\mu, i}\left(J_{1}(\tau), \ldots, J_{d}(\tau), J_{1}\left(\tau^{\prime}\right), J_{i}\left(\tau^{\prime}\right)\right)=0,
$$

so the theorem follows.

\section{Computational details and simplifications for genus two}

We only implemented an algorithm to compute the set of Hilbert modular polynomials in genus 2 , and only for small quadratic fields $K_{0}$, due to the fact that we do not know explicit $q$-expansions for the RM invariants $J_{1}, \ldots, J_{d}$ in any other larger genus. Hence, except where explicitly stated otherwise, we restrict now to the genus 2 case, and for simplicity, we set $d=3$ (recall from Section 5 that this is always theoretically possible). Some of the improvements on the general algorithm Algorithm 6.3 outlined in this section also apply to higher dimension; we will indicate this where relevant.

Lemma 7.2 gives one simplification of the formulae for genus 2: in this case $K_{0}$ is quadratic, so that there is an isomorphism $\mathcal{O}_{K_{0}} \cong \mathcal{O}_{K_{0}}^{\vee}$ of $\mathcal{O}_{K_{0}}$-modules. This isomorphism extends naturally to an isomorphism $\mathrm{SL}_{2}\left(\mathcal{O}_{K_{0}}\right) \backslash\left(K_{0} \otimes \mathbb{H}\right) \cong$ $\mathrm{SL}\left(\mathcal{O}_{K_{0}} \oplus \mathcal{O}_{K_{0}}^{\vee}\right) \backslash\left(K_{0} \otimes \mathbb{H}\right)$. We now work with a compactification of $\mathrm{SL}_{2}\left(\mathcal{O}_{K_{0}}\right) \backslash\left(K_{0} \otimes \mathbb{H}\right)$ instead of $\operatorname{SL}\left(\mathcal{O}_{K_{0}} \oplus \mathcal{O}_{K_{0}}^{\vee}\right) \backslash\left(K_{0} \otimes \mathbb{H}\right)$. Since we do this, in Lemma 4.10, we must replace the matrix group $\Gamma^{0}(\mu)$ with the matrix group $\Gamma^{0}(\mu)^{\prime}$, which we now define.

Definition 7.1. For a totally real number field $K_{0}$ of degree 2 over $\mathbb{Q}$, with ring of integers $\mathcal{O}_{K_{0}}$, and a totally positive element $\mu \in K_{0}$, we define

$$
\Gamma^{0}(\mu)^{\prime}=\left\{\left(\begin{array}{cc}
a & \mu b \\
c & d
\end{array}\right) \in \mathrm{SL}_{2}\left(\mathcal{O}_{K_{0}}\right): a, b, c, d \in \mathcal{O}_{K_{0}}\right\} .
$$


Lemma 7.2. For a totally real number field $K_{0}$ of degree 2 over $\mathbb{Q}$ with ring of integers $\mathcal{O}_{K_{0}}$, and a totally positive element $\mu \in \mathcal{O}_{K_{0}}$ that generates a prime ideal, the set

$$
\mathcal{C}=\left\{\left(\begin{array}{cc}
1 & \omega \\
0 & 1
\end{array}\right): \omega \in \mathcal{O}_{K_{0}} / \mu \mathcal{O}_{K_{0}}\right\} \bigcup\left\{\left(\begin{array}{cc}
0 & 1 \\
-1 & 0
\end{array}\right)\right\}
$$

is a choice of coset representatives for the quotient of groups $\Gamma^{0}(\mu)^{\prime} \backslash \mathrm{SL}_{2}\left(\mathcal{O}_{K_{0}}\right)$.

Proof. The matrix group $\mathrm{SL}_{2}\left(\mathcal{O}_{K_{0}}\right)$ acts on $\mathbb{P}^{1}\left(\mathcal{O}_{K_{0}} / \mu \mathcal{O}_{K_{0}}\right)$ by

$$
\left(\begin{array}{ll}
a & b \\
c & d
\end{array}\right) \cdot(x: y)=(a x+b y: c x+d y) .
$$

Then in particular, the stabilizer of $(0: 1)$ is given by $\Gamma^{0}(\mu)^{\prime}$, and hence by the orbit-stabilizer theorem, there exists a natural bijection from $\mathcal{C}$ to $\Gamma^{0}(\mu)^{\prime} \backslash \mathrm{SL}_{2}\left(\mathcal{O}_{K_{0}}\right)$.

Lemma 7.3. Using the representation of $\Gamma^{0}(\mu)^{\prime} \backslash \mathrm{SL}_{2}\left(\mathcal{O}_{K_{0}}\right)$ given in Lemma 7.2 we can write out explicit $q$-expansions of the coefficients of $\Phi_{\mu}$ and $\Psi_{\mu, i}$ via the following. Let $f$ be a modular form for $\mathrm{SL}_{2}\left(\mathcal{O}_{K_{0}}\right)$ of weight $k$ with $q$-expansion

$$
f(\tau)=\sum_{t \in\left(\mathcal{O}_{K_{0}}^{\vee}\right)^{+}} \alpha(t) e^{2 \pi i \operatorname{tr}(t \tau)}
$$

and let $\ell=\operatorname{Norm}_{K_{0} / \mathbb{Q}}(\mu)$.

1. For $\omega \in \mathcal{O}_{K_{0}} / \mu \mathcal{O}_{K_{0}}$ and $M=\left(\begin{array}{cc}1 & \omega \\ 0 & 1\end{array}\right)$, we have that

$$
\left.f\right|_{\underline{\mu^{-1}} M \tau}=\ell^{-k / 2} \sum_{t \in\left(\mathcal{O}_{K_{0}}^{\vee}\right)^{+}} \zeta_{\ell}^{\operatorname{tr}\left(\ell \mu^{-1} t \omega\right)} \alpha(t) e^{2 \pi i \operatorname{tr}\left(\mu^{-1} t \tau\right)},
$$

where $\left(\mathcal{O}_{K_{0}}^{\vee}\right)^{+}$denotes the totally positive elements of $\mathcal{O}_{K_{0}}^{\vee}$.

2. For $M=\left(\begin{array}{cc}0 & -1 \\ 1 & 0\end{array}\right)$, we have that

$$
\left.f\right|_{\underline{\mu^{-1}} M \tau}=\ell^{k / 2} \sum_{t \in\left(\mathcal{O}_{K_{0}}^{\vee}\right)^{+}} \alpha(t) e^{2 \pi i \operatorname{tr}(\mu t \tau)},
$$

where $\left(\mathcal{O}_{K_{0}}^{\vee}\right)^{+}$denotes the totally positive elements of $\mathcal{O}_{K_{0}}^{\vee}$.

Proof. Follows immediately from the definitions.

Remark 7.4. In any dimension $g$, if $K_{0}$ has class number 1 then $\mathcal{O}_{K_{0}} \cong \mathcal{O}_{K_{0}}^{\vee}$ as $\mathcal{O}_{K_{0}}^{\vee}$-modules, so Lemma 7.2 and Lemma 7.3 would also hold in this case. 
Algorithm 6.3 is extremely slow and uses a lot of memory. Although the resulting coefficients of the set of Hilbert modular polynomials are relatively small, the sizes of the numbers appearing in the intermediary linear algebra computations on the Fourier expansions blow up very quickly (to several orders of magnitude larger than the sizes occuring in the output). The output values are only small because they are coefficients of Hilbert modular forms and so 'special' (intuitively speaking). To this end, we give here some practical improvements on the computation time and memory usage. First of all, we do not compute the third modular polynomial $H_{\mu, 3}\left(X_{1}, X_{2}, X_{3}, Y, Z_{3}\right)$; Algorithm 7.5 shows that, given $(\mathrm{A}, \xi, \iota) \in \mathbf{P O r d}_{\mathbb{C}, K_{0}}$, we can compute every abelian surface $\mu$-isogenous to it without using $H_{\mu, 3}$.

\section{Algorithm 7.5.}

INPUT: The first 2 Hilbert modular polynomials $G_{\mu}\left(X_{1}, X_{2}, X_{3}, Y\right)$ and $H_{\mu, 2}\left(X_{1}, X_{2}, X_{3}, Y, Z_{2}\right)$, as defined in Definition 1.6 the RM isomorphism invariants $\left(j_{1}, j_{2}, j_{3}\right) \in \mathbb{C}^{3}$ of some $(\mathrm{A}, \xi, \iota) \in \mathbf{P O r d}_{\mathbb{C}, K_{0}}$, as defined in Definition 1.2. and the minimal polynomial $m(X) \in \mathbb{Q}\left(J_{1}, J_{2}\right)[X]$ of $J_{3}$, as in Section 5 .

OUTPUT: The RM isomorphism invariants of each $\left(\mathrm{A}^{\prime}, \xi^{\prime}, \iota^{\prime}\right) \in \mathbf{P O r d}_{\mathbb{C}, K_{0}}$ that is $\mu$-isogenous to $(\mathrm{A}, \xi, \iota)$, or failure.

1. Set $L$ to be the list of the $\operatorname{Norm}_{K_{0} / \mathbb{Q}}(\mu)+1$ roots of $G_{\mu}\left(j_{1}, j_{2}, j_{3}, Y\right)$. If the roots are not distinct, output failure.

2. For every $j_{1}^{\prime} \in L$ :

(a) set $j_{2}^{\prime}$ to be the unique element of $\mathbb{C}$ for which $H_{\mu, 2}\left(j_{1}, j_{2}, j_{3}, j_{1}^{\prime}, j_{2}^{\prime}\right)=$ 0 ,

(b) set $L_{0}$ to be the list of the roots of $m(X)$ evaluated at $\left(J_{1}, J_{2}\right)=$ $\left(j_{1}^{\prime}, j_{2}^{\prime}\right)$.

(c) for every $l \in L_{0}$, check if $G_{\mu}\left(j_{1}^{\prime}, j_{2}^{\prime}, l, j_{1}\right)=0$. If true for exactly one $l$, set $j_{3}^{\prime}=l$. Else, output failure.

(d) add $\left(j_{1}^{\prime}, j_{2}^{\prime}, j_{3}^{\prime}\right)$ to list $L^{\prime}$.

3. Return $L^{\prime}$.

Remark 7.6. An analogous algorithm to Algorithm 7.5 in arbitrary dimension $g$ and $d \geq g$ RM isomorphism invariants allows us to compute only $g$ polynomials in place of $d$.

Remark 7.7. Heuristically, we expect that for large primes $p$ and most $(\mathrm{A}, \xi)$ and $\left(\mathrm{A}^{\prime}, \xi^{\prime}\right) \in \mathbf{P O r d}_{\mathbb{F}_{p}, K_{0}}$, there exists a $\mu$-isogeny $(\mathrm{A}, \xi) \rightarrow\left(\mathrm{A}^{\prime}, \xi^{\prime}\right)$ if and only if

$$
\begin{aligned}
& G_{\mu}\left(J_{1}(\mathrm{~A}), J_{2}(\mathrm{~A}), J_{3}(\mathrm{~A}), J_{1}\left(\mathrm{~A}^{\prime}\right)\right) \\
\equiv & H_{\mu, 1}\left(J_{1}(\mathrm{~A}), J_{2}(\mathrm{~A}), J_{3}(\mathrm{~A}), J_{1}\left(\mathrm{~A}^{\prime}\right), J_{2}\left(\mathrm{~A}^{\prime}\right)\right) \\
\equiv & 0 \bmod p
\end{aligned}
$$

and $J_{3}\left(\mathrm{~A}^{\prime}\right)$ is the same as the output of Step 2 of Algorithm 7.5 (with $\mathbb{C}$ replaced by $\mathbb{F}_{p}$ ) with

$$
\left(j_{1}, j_{2}, j_{3}, j_{1}^{\prime}, j_{2}^{\prime}\right)=\left(J_{1}(\mathrm{~A}), J_{2}(\mathrm{~A}), J_{3}(\mathrm{~A}), J_{1}\left(\mathrm{~A}^{\prime}\right), J_{2}\left(\mathrm{~A}^{\prime}\right)\right) .
$$


If we assume that the heuristics of Remark 7.7 then we have a second major practical improvement: do computations in finite fields in place of in $\mathbb{Q}$ and $\mathbb{Q}\left(\zeta_{\mathrm{Norm}_{K_{0} / \mathbb{O}}(\mu)}\right)$ and then use the Chinese Remainder Theorem. This idea is used in Algorithm 7.8.

One advantage of working over a finite field in place of $\mathbb{Q}$ is that while the algorithm is running over $\mathbb{Q}$, the coefficients of the $q$-expansions blow up, using up memory space and slowing down computations, so that Algorithm 7.8 is significantly faster than Algorithm 6.3. A major disadvantage however is that we do not currently have the tools to compute useful input values $B, D$, and $p_{0}$. The only method available, to the best knowledge of the author, is to make an educated guess (based on the specific RM isomorphism being used and smaller already computed examples), and then check correctness in one of the following two ways:

1. Find a genus 2 curve $C$ whose Jacobian $\mathcal{J}(C)$ has maximal complex multiplication by a CM-field $K$ with maximal totally real subfield $K_{0}$, for example by referring to Kohel's database [17. Choose a prime $p>2 B D$ and let $\mathcal{J}(C)_{p}$ be the reduction of $\mathcal{J}(C) \bmod p$. Compute the shape of the $\mu$ and $\sigma(\mu)$-isogeny graph containing $\mathcal{J}(C)_{p}$ using [20, Volcano Theorem, Chapter 3]. Compute the RM-isomorphism invariants $\left(J_{1}, J_{2}, J_{3}\right)$ of $\mathcal{J}(C)_{p}$ using (something similar to) Example 5.4-note that there are two choices for $J_{3}$. Re-compute the shape of the $\mu$-isogeny and $\sigma(\mu)$-isogeny graphs using the set of Hilbert modular polynomials (the two choices for $J_{3}$ correspond to the two conjugates of $\mu$ ). If the graphs do not agree, then the algorithm failed and the input values $B, D$, and $p_{0}$ should be increased. Else, repeat the correctness check e.g. 100 times to reach some level of certainty that the set of Hilbert modular polynomials are in fact correct.

2. Find the RM-isomorphism invariants $\left(J_{1}, J_{2}, J_{3}\right)$ (up to choice for $J_{3}$ ) of a genus 2 curve whose Jacobian has maximal real multiplication for example as described in the previous point, and check that $G_{\mu}\left(J_{1}, J_{2}, J_{3}, Y\right)$ factors as expected given [2, Proposition 3.13] (for one choice of $J_{3}$ ). If it does not factor correctly, then the algorithm failed and the input values should be increased. Else, repeat the correctness check as many times as required for the desired probability of correctness.

\section{Algorithm 7.8. \\ INPUT:}

1. A totally real number field $K_{0}$ of degree 2 over $\mathbb{Q}$.

2. The $q$-expansions of generators $\gamma_{1}, \ldots, \gamma_{s} \in \mathcal{M}_{K_{0}}(\mathbb{Z})$ of the $\mathbb{Q}$-algebra $\mathcal{M}_{K_{0}}(\mathbb{Q})$.

3. The images of $\gamma_{1}, \ldots, \gamma_{s}$ under $\rho$ as rational functions of $J_{1}, J_{2}, J_{3}$, where $\rho$ is as defined in 19 .

4. A totally positive element $\mu \in K_{0}$ that generates a prime ideal.

5. An upper bound $B$ on the absolute values and a common denominator $D$ of the rational coefficients of the coefficients of $\Phi_{\mu}(Y)$ and $\Psi_{\mu, 2}\left(Y, Z_{2}\right)$ when represented as formal polynomials $\gamma_{1}, \ldots, \gamma_{s}$. 
6. A prime $p_{0}$ such that for every prime $p \geq p_{0}$, the $q$-expansion coefficients in Step 1 of Algorithm 6.3 have denominator coprime to $p$, and when replacing $\mathbb{Q}$ and $\mathbb{Q}\left(\zeta_{\ell}\right)$ by $\mathbb{F}_{p}$ and $\mathbb{F}_{p}\left(\zeta_{\ell}\right)$, the system of linear equations in Step 2 of Algorithm 6.3 still has a unique solution.

OUTPUT: The first 2 polynomials

$$
\begin{aligned}
G_{\mu}\left(X_{1}, X_{2}, X_{3}, Y\right) & \in \mathbb{Z}\left[X_{1}, X_{2}, X_{3}, Y\right], \text { and } \\
H_{\mu, 2}\left(X_{1}, X_{2}, X_{3}, Y, Z_{2}\right) & \in \mathbb{Z}\left[X_{1}, X_{2}, X_{3}, Y, Z_{2}\right]
\end{aligned}
$$

of Definition 1.6.

1. Create a list $L$ of primes in the following way:

(a) Set $i=0$.

(b) Set $b=p_{i}$.

(c) Set $p_{i+1}=\min \left\{n \in \mathbb{Z}_{>b}: n\right.$ prime, $\left.n \equiv 1 \bmod \operatorname{Norm}_{K_{0} / \mathbb{Q}}(\mu)\right\}$. (This condition is to speed up the computations as the $\operatorname{Norm}_{K_{0} / \mathbb{Q}}(\mu)^{\text {th }}$ roots of unity are then in $\mathbb{F}_{p}$.)

(d) Reduce the coefficients of the $q$-expansions of $\gamma_{1}, \ldots, \gamma_{s} \bmod p_{i+1}$ to get

$$
\overline{\gamma_{1}}, \ldots, \overline{\gamma_{s}} \in \mathcal{M}_{K_{0}}(\mathbb{Z}) / p_{i+1} \mathcal{M}_{K_{0}}(\mathbb{Z}) .
$$

If $\overline{\gamma_{1}}, \ldots, \overline{\gamma_{s}}$ generate $\mathcal{M}_{K_{0}}(\mathbb{Z}) / p_{i+1} \mathcal{M}_{K_{0}}(\mathbb{Z})$ as a $\mathbb{F}_{p_{i+1}}$-algebra, go to step (e). Else, set $b=p_{i+1}$ and go to step (c).

(e) If $\prod_{j=1}^{i+1} p_{j}<2 B D$ then set $i=i+1$ and go to (b). Else return

$$
L=\left\{p_{1}, \ldots, p_{i+1}\right\} .
$$

2. Write the coefficients mod $p$ of $\Phi_{\mu}(Y)$ and $\Psi_{\mu, 2}(Y)$ as formal polynomials in $\gamma_{1}, \ldots, \gamma_{s}$ for every $p \in L$ by following Step 1 and 2 of Algorithm 6.3. with $\mathbb{Q}\left(\right.$ and $\left.\mathbb{Q}\left(\zeta_{\mathrm{Norm}_{K_{0} / \mathbb{Q}}}\right)\right)$ replaced by $\mathbb{F}_{p}$. (This can be done in parallel.)

3. Use the Chinese Remainder Theorem to compute the coefficients of $D \Phi_{\mu}(Y)$ and $D \Psi_{\mu, 2}(Y)$ as formal polynomials in $\gamma_{1}, \ldots, \gamma_{s}$ with integer coefficients.

4. Compute $G_{\mu}$ and $H_{\mu, 2}$ following Step 3 of Algorithm 6.3 .

Remark 7.9. Under much stronger heuristics it may also be possible to generalise Algorithm 7.5 to arbitrary dimension. More specifically, the heuristics of Remark 7.7 rely somewhat on the fact that our RM isomorphism invariants in genus 2 are pullbacks of Igusa invariants, which are known to respect this property. Furthermore, any kind of correctness check on the input values $B, D$, and $p_{0}$ like those described above relies on finding some principally polarised abelian varieties with maximal complex multiplication by a CM-field $K$ that has $K_{0}$ as a maximal totally real subfield. This construction is commonly known as the 'CM-method', and to the knowledge of the author there is little known about how to do this in arbitrary dimension. 
As mentioned above, the major disadvantage of Algorithm 7.8 is that it is heuristic. However, the speed up is quite significant: for $\operatorname{Norm}_{K_{0} / \mathbb{Q}}(\mu)=$ 11, Algorithm 6.3 took 1 week and Algorithm 7.8 took 90 minutes (on the same machine). Also, we can check the output by looking at the behaviour of the polynomials as described above. Even with these improvements, there is still a long way to go before this algorithm is practical for larger values of $\operatorname{Norm}_{K_{0} / \mathbb{Q}}(\mu)$; Table 1 gives the timings for the computations that we have done so far. Note that Table 1 also includes one entry for $K_{0}=\mathbb{Q}(\sqrt{2})$-for this case we have an implementation that works currently only for the case $\mu=\sqrt{2}$ (this implementation uses only $J_{1}$ and $J_{2}$ ). It should be possible to generalise this implementation to other values of $\mu \in \mathbb{Q}(\sqrt{2})$ using the formulae for the non-symmetric part $J_{3}$ of RM isomorphism invariant presented in [25]; however, so far attempts have not yielded results and we leave this to future work.

\begin{tabular}{|r|c|c|c|c|c|c|}
\hline $\operatorname{Disc}\left(K_{0}\right)$ & 8 & 5 & 5 & 5 & 5 & 5 \\
\hline $\mathrm{N}_{K_{0} / \mathbb{Q}}(\mu)$ & 2 & 4 & 5 & 9 & 11 & 19 \\
\hline Time & $2 \mathrm{~s}$ & $63 \mathrm{~s}$ & $90 \mathrm{~s}$ & $\sim 4 \mathrm{~m}$ & $\sim 90 \mathrm{~m}$ & $\sim 3 \mathrm{~d}$ \\
\hline
\end{tabular}

Table 1: Timings for computation of Hilbert modular polynomials ${ }^{1}$

Remark 7.10. Choosing the representations of the invariants in such a way to minimise the coefficients of the Hilbert modular polynomials would give a very siginificant speed-up, especially taking into account Algorithm 7.8. We leave this for furture work.

Remark 7.11. Dudeanu, Jetchev, Robert, and Vuille [9] have presented an algorithm to compute a $\mu$-isogeny directly from its kernel. When the isogeny class of a given $(\mathrm{A}, \xi, \iota) \in \mathbf{P O r d}_{\mathbb{F}_{p}, K_{0}}$ is large enough, it should be possible to determine the coefficients of the Hilbert modular polynomial modulo $p$ by looking at a large set of roots (found using the algorithm of [9]) and applying linear algebra. In combination with the Chinese remainder theorem, under the heuristics of Remark 7.7, this could give an alternative (probably also heuristic) algorithm to compute Hilbert modular polynomials. Whether or not such an algorithm would be more efficient than Algorithm 7.5 is of course subject to careful complexity analysis. We leave this for future work.

\section{Applications}

There are many potential applications for these polynomials, some of which have already been explored. We give three of these applications below.

\footnotetext{
${ }^{1}$ These timings are using the fastest algorithm in this paper, Algorithm 7.8 although it is only conjecturally correct.
} 


\subsection{Point counting}

One natural application is a generalisation of the Schoof-Elkies-Aktin pointcounting algorithm to genus 2 curves. Schoof 30 gave an algorithm to count points on elliptic curves over finite fields in polynomial time. Schoof's algorithm was improved by Atkin using factorisations of modular polynomials, and later improved further by Elkies. Although there already exists a theoretical polynomial-time algorithm to count points on genus 2 curves over finite fields due to Pila [27] and a practical polynomial-time algorithm to count points on genus 2 curves over finite fields with real multiplication due to Gaudry, Kohel, and Smith [13, it is natural to study the factorisation patterns of the Hilbert modular polynomials presented in this paper to attempt to generalise Atkin's improvements to Schoof's algorithm to genus 2 curves with maximal real multiplication. The genus-two-maximal-real-multiplication version of Schoof, Elkies, and Atkin's point counting algorithm using the set of Hilbert modular polynomials given in this paper has in fact already been studied by Ballentine, Guillevic, Lorenzo-Garcia, Massierer, Martindale, Smith, and Top [2]; the asymptotic complexity gives a significant improvement on Pila's method and a small improvement on Gaudry, Kohel, and Smith's method, although this modular polynomial method is of course very restrictive compared to either pre-exisiting method as it currently only applies in practise to curves with maximal real multiplication by $\mathbb{Q}(\sqrt{5})$.

\subsection{Walking on isogeny graphs}

Another natural application is the 'navigation' of isogeny graphs of genus 2 curves. There has been a growing interest in the isogeny graphs of elliptic curves over finite fields recently due to the development of isogeny-based cryptography [12, 8, 7]. Every such protocol relies on the ability to compute 'neighbours' in an $\ell$-isogeny graph, that is, given an elliptic curve $E / \mathbb{F}_{q}$, to compute all the elliptic curves that are $\ell$-isogenous to $E$ (up to isomorphism). In the case of elliptic curves, there are many different options for doing this, such as Vélu's formulas [34], or division polynomials in combination with Kohel's algorithm [18, Section 2.4], or modular polynomials in combination with Kohel's algorithm. Modular polynomials are rarely the most efficient option, although for some applications they do prove to be the best choice, e.g. [3, Appendix D].

There is a case for attempting to generalise these protocols to genus 2 , especially given the recent research [29] suggesting that genus 2 arithmetic can be more efficient than elliptic curve arithmetic. The protocol presented in [8] should generalise to genus 2 curves with maximal real multiplication directly-given that the structure of isogeny graphs for principally polarised simple ordinary abelian surfaces with maximal real multiplication is the same as for ordinary elliptic curves defined over $\mathbb{F}_{q}[20,5]$. In order to actually implement such a protocol, there has to be a method of navigating the isogeny graphs, that is, of computing all the surfaces that are $\mu$-isogenous to a given surface. Dudeanu, Jetchev, Robert, and Vuille 9] have presented an algorithm

to compute a $\mu$-isogeny from its kernel as an ideal in the endomorphism ring; 
this can be thought of as a genus 2 analogue of Vélu's formulas. The Hilbert modular polynomials presented in this paper give another method for computing neighbours in the $\mu$-isogeny graph. The Hilbert modular polynomial method is currently more viable for small $\mu$ with $K_{0}=\mathbb{Q}(\sqrt{5})$ and large (cryptographic size) $p$.

\subsection{Computing class polynomials}

A third natural application is the generalisation of Sutherland's algorithm for computing Hilbert class polynomials 32 to genus 2. A Hilbert class polynomial over a field $k$ for a given maximal order $\mathcal{O}$ in a imaginary quadratic field is a polynomial whose roots are given by the $j$-invariants of all the elliptic curves over $k$ for which the endomorphism ring is isomorphism to $\mathcal{O}$. Via these polynomials it is possible to construct an elliptic curve over $\mathbb{F}_{p}$ with a given number of points. Sutherland [32] gives a method to compute these polynomials using modular polynomials, which should generalise in a natural way to genus 2 using the polynomials presented in this paper. We leave the details to future work.

\section{Acknowledgements}

The author would like to thank her PhD supervisor, Marco Streng, for suggesting this research topic and for invaluable input and guidance. Thank you also to the anonymous referee for their careful reading and helpful comments. Sections 1-6 of this article also appear as Chapter 2 and relevant parts of Chapter 1 of the $\mathrm{PhD}$ thesis of the author [20, which was completed at Universiteit Leiden and Université de Bordeaux with the support of funding from the ALGANTdoc programme. This work was also partly supported by the Netherlands Organisation for Scientific Research (NWO) under CHIST-ERA USEIT (grant number 651.002.004). 


\section{References}

[1] W.L. Jr. Baily and A. Borel. On the compactification of arithmetically defined quotients of bounded symmetric domains Bull. Amer. Math. Soc., 70:588-593, 1964.

[2] S. Ballentine, A. Guillevic, E. Lorenzo-García, M. Massierer, C. Martindale, B. Smith, and J. Top. Isogenies for point counting on genus two hyperelliptic curves with maximal real multiplication. In Algebraic Geometry for Coding Theory and Cryptography, volume 9 of Association for Women in Mathematics Series, pages 63-94. Springer Int. Pub., 2017.

[3] D. Bernstein, T. Lange, C. Martindale, and L. Panny. Quantum circuits for the CSIDH: optimizing quantum evaluation of isogenies. EUROCRYPT 2019, 2018.

[4] Wieb Bosma, John Cannon, and Catherine Playoust. The Magma algebra system. I. The user language. J. Symbolic Comput., 24(3-4):235-265, 1997. Computational algebra and number theory (London, 1993).

[5] E.H. Brooks, D. Jetchev, and B. Wesolowski. Isogeny graphs of ordinary abelian varieties. Research in Number Theory, 3, 2017.

[6] R. Bröker and K. Lauter. Modular polynomials for genus 2. LMS Journal of Computation and Mathematics, 12:326-339, 2009.

[7] W. Castryck, T. Lange, C. Martindale, L. Panny, and J. Renes. CSIDH: An efficient post-quantum commutative group action. ASIACRYPT 2018, 2018.

[8] L. De Feo, J. Kieffer, and B. Smith. Towards practical key exchange from ordinary isogeny graphs. ASIACRYPT 2018, 2018.

[9] A. Dudeanu, D. Jetchev, D. Robert, and M. Vuille. Cyclic Isogenies for Abelian Varieties with Real Multiplication, 2017.

[10] R. Dupont. Moyenne Arithmético-géométrique, Suites de Borchardt et Applications. PhD thesis, Ecole Polytechnique, 2006.

[11] A. Enge. Computing modular polynomials in quasi-linear time. Math. Comp., 78(267):1809-1824, 2009.

[12] L. De Feo, D. Jao, and J. Plût. Towards quantum-resistant cryptosystems from supersingular elliptic curve isogenies. Journal of Mathematical Cryptology, 8(3):209-247, 2014.

[13] P. Gaudry, D. Kohel, and B. Smith. Counting points on genus 2 curves with real multiplication. In Advances in cryptology-ASIACRYPT 2011, volume 7073 of Lecture Notes in Comput. Sci., pages 504-519. Springer, Heidelberg, 2011. 
[14] K.-B. Gundlach. Die Bestimmung der Funktionen zur Hilbertschen Modulgruppe des Zahlkörpers $Q(\sqrt{ } 5)$, Math. Ann., 152:226-256, 1963.

[15] R. Hartshorne. Algebraic geometry. Springer-Verlag, New York-Heidelberg, 1977. Graduate Texts in Mathematics, No. 52.

[16] J. Igusa. Arithmetic variety of moduli for genus two. Ann. of Math. (2), 72:612-649, 1960.

[17] D. Kohel. The echidna database. https://www.i2m.univ-amu.fr/perso/ david.kohel/dbs/index.html.

[18] D. Kohel. Endomorphism rings of elliptic curves over finite fields. $\mathrm{PhD}$ thesis, University of California, Berkeley, 1996.

[19] K. Lauter, M. Naehrig, and T. Yang. Hilbert theta series and invariants of genus 2 curves. J. Number Theory, 161:146-174, 2016.

[20] C. Martindale. Isogeny Graphs, Modular Polynomials, and Applications. $\mathrm{PhD}$ thesis, Universiteit Leiden and Université de Bordeaux, 2018. http: //www.martindale.info/research/Thesis.pdf

[21] S. Mayer. Hilbert Modular Forms for the Fields $Q(\sqrt{5}), Q(\sqrt{13})$ and $Q(\sqrt{17})$. $\mathrm{PhD}$ thesis, Rheinisch-Westfälischen Technischen Hochschule Aachen, 2007.

[22] E. Milio. Calcul de polynômes modulaires en dimension 2. $\mathrm{PhD}$ thesis, Université de Bordeaux, 2015. https://www.theses.fr/191225460.

[23] E. Milio. A quasi-linear time algorithm for computing modular polynomials in dimension 2. LMS J. Comput. Math., 18(1):603-632, 2015.

[24] E. Milio and D. Robert. Modular polynomials on Hilbert surfaces. 2017. https://hal.archives-ouvertes.fr/hal-01520262v2/document.

[25] R. Mueller. Hilbertsche Modulformen und Modulfunktionen zu Q $(\sqrt{8})$. Math. Ann., 266(1):83-103, 1983.

[26] R. Mueller. Hilbertsche Modulformen und Modulfunktionen zu Q $(\sqrt{5})$. Arch. Math. (Basel), 45(3):239-251, 1985.

[27] J. Pila. Frobenius maps of abelian varieties and finding roots of unity in finite fields. Math. Comp., 55(192):745-763, 1990.

[28] M. Rapoport. Compactifications de l'espace de modules de HilbertBlumenthal. Compositio Math., 36(3):255-335, 1978.

[29] J. Renes, P. Schwabe, B. Smith, and L. Batina. $\mu$ Kummer: efficient hyperelliptic signatures and key exchange on microcontrollers. 2016. https: //doi.org/10.1007/978-3-662-53140-2_15.

[30] R. Schoof. Counting points on elliptic curves over finite fields. J. Théor. Nombres Bordeaux, 7(1):219-254, 1995. 
[31] A. Sutherland. Modular polynomials, 2018. https://math.mit.edu/ rdrew/ClassicalModPolys.html.

[32] A.V. Sutherland. Computing Hilbert class polynomials with the Chinese remainder theorem. Math. Comp., 80(273):501-538, 2011.

[33] G. van der Geer. Hilbert modular surfaces, volume 16 of Ergebnisse der Mathematik und ihrer Grenzgebiete (3) [Results in Mathematics and Related Areas (3)]. Springer-Verlag, Berlin, 1988.

[34] J. Vélu. Isogénies entre courbes elliptiques. Comptes Rendus de l'Académie des Sciences de Paris, 273:238-241, 1971.

[35] D. Zagier. Elliptic modular forms and their applications. https://people.mpim-bonn.mpg.de/zagier/files/doi/10.1007/

978-3-540-74119-0_1/fulltext.pdf. 